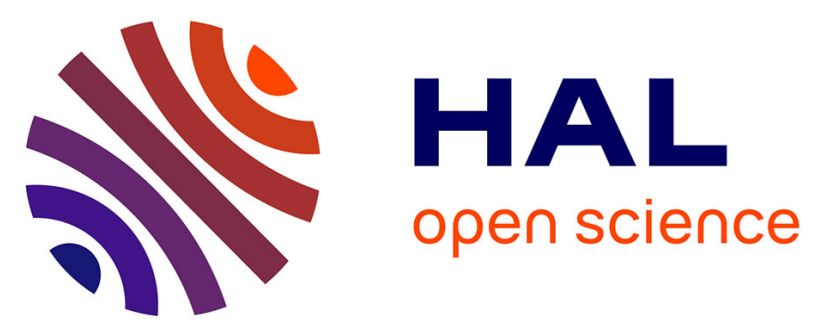

\title{
Effects of chronic exposure to cadmium and temperature, alone or combined, on the threespine stickleback ( Gasterosteus aculeatus ): interest of digestive enzymes as biomarkers
}

Younes M.I. Hani, Cyril Turies, Olivier Palluel, Laurence Delahaut, Véronique Gaillet, Anne Bado-Nilles, Jean-Marc Porcher, Alain Geffard, Odile Dedourge-Geffard

\section{- To cite this version:}

Younes M.I. Hani, Cyril Turies, Olivier Palluel, Laurence Delahaut, Véronique Gaillet, et al.. Effects of chronic exposure to cadmium and temperature, alone or combined, on the threespine stickleback ( Gasterosteus aculeatus ): interest of digestive enzymes as biomarkers. Aquatic Toxicology, 2018, 199, pp.252-262. 10.1016/j.aquatox.2018.04.006 . hal-01767659

\section{HAL Id: hal-01767659 \\ https://hal.science/hal-01767659}

Submitted on 6 Mar 2019

HAL is a multi-disciplinary open access archive for the deposit and dissemination of scientific research documents, whether they are published or not. The documents may come from teaching and research institutions in France or abroad, or from public or private research centers.
L'archive ouverte pluridisciplinaire HAL, est destinée au dépôt et à la diffusion de documents scientifiques de niveau recherche, publiés ou non, émanant des établissements d'enseignement et de recherche français ou étrangers, des laboratoires publics ou privés. 


\section{Accepted Manuscript}

Title: Effects of chronic exposure to cadmium and temperature, alone or combined, on the threespine stickleback (Gasterosteus aculeatus): interest of digestive enzymes as biomarkers

Authors: Younes Mohamed Ismail Hani, Cyril Turies, Olivier Palluel, Laurence Delahaut, Véronique Gaillet, Anne Bado-nilles, Jean-Marc Porcher, Alain Geffard, Odile Dedourge-geffard

$\begin{array}{ll}\text { PII: } & \text { S0166-445X(18)30346-1 } \\ \text { DOI: } & \text { https://doi.org/10.1016/j.aquatox.2018.04.006 } \\ \text { Reference: } & \text { AQTOX 4914 }\end{array}$

To appear in: $\quad$ Aquatic Toxicology

Received date: $\quad$ 21-11-2017

Revised date: $\quad 1-4-2018$

Accepted date: $\quad 10-4-2018$

Please cite this article as: Hani, Younes Mohamed Ismail, Turies, Cyril, Palluel, Olivier, Delahaut, Laurence, Gaillet, Véronique, Bado-nilles, Anne, Porcher, JeanMarc, Geffard, Alain, Dedourge-geffard, Odile, Effects of chronic exposure to cadmium and temperature, alone or combined, on the threespine stickleback (Gasterosteus aculeatus): interest of digestive enzymes as biomarkers.Aquatic Toxicology https://doi.org/10.1016/j.aquatox.2018.04.006

This is a PDF file of an unedited manuscript that has been accepted for publication. As a service to our customers we are providing this early version of the manuscript. The manuscript will undergo copyediting, typesetting, and review of the resulting proof before it is published in its final form. Please note that during the production process errors may be discovered which could affect the content, and all legal disclaimers that apply to the journal pertain. 
Effects of chronic exposure to cadmium and temperature, alone or combined, on the threespine stickleback (Gasterosteus aculeatus): interest of digestive enzymes as biomarkers.

\section{Authors}

Younes Mohamed Ismail HANI ${ }^{1,2}$, Cyril TURIES ${ }^{1}$, Olivier PALLUEL ${ }^{1}$, Laurence DELAHAUT $^{2}$, Véronique GAILLET ${ }^{2}$, Anne BADO-NILLES ${ }^{1}$, Jean-Marc PORCHER ${ }^{1}$, Alain GEFFARD $^{2}$, Odile DEDOURGE-GEFFARD ${ }^{2 *}$

1 Institut National de l'Environnement Industriel et des Risques (INERIS), UMR-I O2 SEBIO (Stress Environnementaux et Biosurveillance des milieux aquatiques), Verneuil-en-Halatte, France.

2 Université de Reims Champagne-Ardenne (URCA), UMR-I 02 SEBIO (Stress Environnementaux et Biosurveillance des milieux aquatiques), Moulin de la Housse, Reims, France.

*Corresponding author:

\section{Dr. Odile DEDOURGE-GEFFARD}

Université de Reims Champagne-Ardenne (URCA), UMR-I 02 SEBIO (Stress Environnementaux et Biosurveillance des milieux aquatiques)

Moulin de la Housse, B.P. 1039, 51687 Reims, France.

Tel. : +33 (0) 326913328

E-mail : odile.dedourge@univ-reims.fr 


\title{
Highlights
}

- Chronic effects of cadmium and /or temperature were studied.

- Temperature alone impacted antioxidant and energy reserve parameters.

- Digestive enzymes were negatively impacted by the both stress. .

- The negative effect of cadmium was higher and earlier associated with temperature increase.

- Digestive enzymes appear as potential biomarkers of organism's health.

\begin{abstract}
The development of predictive, sensitive and reliable biomarkers is of crucial importance for aquatic biomonitoring to assess the effects of chemical substances on aquatic organisms, especially when it comes to combined effects with other stressors (e.g. temperature). The first purpose of the present study was to evaluate the single and combined effects of 90 days of exposure to an environmental cadmium concentration $\left(0.5 \mu \mathrm{g} . \mathrm{L}^{-1}\right)$ and two water temperatures $\left(16\right.$ and $21{ }^{\circ} \mathrm{C}$ ) on different parameters. These parameters are involved in (i) the antioxidant system (superoxide dismutase activity - SOD - and total glutathione levels - GSH -), (ii) the energy metabolism, i.e. energy reserves (glycogen, lipids, proteins) and digestive enzymes (trypsin, amylase, intestinal alkaline phosphatase - IAP -), and (iii) biometric parameters (weight, length, Fulton's condition factor, and the gonadosomatic index - GSI -) of threespine stickleback (Gasterosteus aculeatus). The second purpose was to determine the interest of the three digestive enzymes as biomarkers in comparison with the other parameters. The higher temperature $\left(21^{\circ} \mathrm{C}\right)$ impacted the anti-oxidant and energy reserve parameters. In liver, GSH levels increased on day 60, while SOD decreased on days 15 and 90, with a significant decrease of protein and lipid energy reserves on day 90. In muscle, the higher temperature decreased SOD activity only on day 90. G. aculeatus biometric parameters were also impacted by the higher temperature, which limited stickleback growth after 90 days of exposure. In female sticklebacks, the GSI peaked on day 60 and decreased sharply on day 90, while the highest values were reached at day 90 in the control groups, suggesting impaired reproduction in sticklebacks raised at $21{ }^{\circ} \mathrm{C}$. These results suggest that $21{ }^{\circ} \mathrm{C}$ is an upper-limit temperature for
\end{abstract}


long-term physiological processes in sticklebacks. In contrast, very low-concentration cadmium exposure had no effect on classical biomarkers (energy reserves, antioxidant parameters, biometric parameters). However, digestive enzymes showed an interesting sensitivity to cadmium, which was emphasized by high temperature. The activity of the three digestive enzymes decreased significantly on day 90 when sticklebacks were exposed to cadmium alone, while the decrease was stronger and was recorded earlier (from day 15) when they were exposed to the cadmium-temperature combination. Compared to conventional measurements, digestive enzymes responded rapidly. This could be an important advantage for them to be used as early warning tools to reflect the health status of organisms, particularly for trypsin and IAP activities.

Keywords: antioxidant parameters; digestive enzymes; cadmium stress; energy metabolism; Gasterosteus aculeatus; temperature stress.

\section{Introduction}

The water quality of aquatic ecosystems can be assessed using different methods based on chemical and ecological approaches. With over 130 million chemical substances known to date (CAS, 2017), the development of predictive, sensitive and reliable tools to assess the impacts of chemicals is of crucial importance for the biomonitoring of aquatic ecosystems. Among them, biomarkers have occupied an important place in ecotoxicology in the last decades (Forbes et al., 2006). In this context, energy metabolism parameters have emerged as predictable and useful biomarkers to detect the effects of chemicals on different aquatic organisms (De Coen and Janssen, 1997; Charron et al., 2013; Pi et al., 2016). As energy is the most determining biological parameter involved in all the physiological processes of living beings, disturbances of the energy metabolism appear to be a good indicators of the general health status of organisms (De Coen and Janssen, 2003).

Different biological parameters related to the energy metabolism are used in ecotoxicology. They reflect a response to a stress condition, which can be expressed at different levels of living organisms, from macromolecular to individual levels, e.g. the adenylate energy charge (AEC), energy reserves (glycogen, lipids, proteins), cellular energy allocation (CEA), serum indicators (glucose, cortisol), condition indices (Fulton's condition factor, the hepatosomatic index). The usefulness of these parameters has been demonstrated in different aquatic organisms, including fish (Dickson et al., 1982; Soengas et al., 1996; Sancho et al., 1998; Almeida et al., 2001; Huntingford et al., 2001; Levesque et al., 2002; Pi et al., 2016). Other parameters representing a crucial step in the energy acquisition stage, i.e. digestive 
enzymes, have recently been used as health status indicators in aquatic invertebrates (gammarids and daphnia), as well as in fish. In fish, three possible primary sites for pollutant uptake are the body surface, the gills, and the alimentary canal (Perera et al., 2015). Since the latter acts as a secretion site of digestive enzymes, exposure to pollutants could disturb digestive enzyme activity. Hence, several authors have suggested using these parameters as potential biomarkers in ecotoxicology (Dedourge-Geffard et al., 2009; Wang et al., 2015; Caruso et al., 2016). Under stressful situations, energy allocation in fish is disturbed because a large proportion of ingested energy is invested in detoxification, involving the cellular antioxidant defence system: superoxide dismutase (SOD), total glutathione (GSH), glutathione reductase (GR), catalase (CAT), etc. These parameters are also used as classical biomarkers in aquatic biomonitoring, indicating the level of exposure of an organism to chemical substances such as metals or pesticides (Cossu et al., 2000; Sanchez et al., 2007).

Aquatic contamination results from both organic and inorganic pollutants (mainly metals). But in natural ecosystems, living organisms are subjected not only to anthropogenic chemical stresses, but also to multiple stressors, whether natural or introduced into the environment by humans. Temperature is a potent physical stressor and a key factor in the control of the fundamental physiological processes involved in survival, growth, and reproduction of ectotherm organisms (Heugens et al., 2001). The water temperature often fluctuates naturally due to diurnal and seasonal variations that result in extremely low or high temperatures that can be harmful or even fatal to living aquatic organisms (Heugens et al., 2001). Similarly, with the anthropogenic climate change, more extreme high temperature variations can be expected (IPCC, 2013). Although temperature scenarios can be simulated from carbon dioxide emission models (IPCC, 2013), predicting the biological effects of multiple stressors on living beings remains difficult, and even impossible with the current state of knowledge. When it comes to xenobiotics and temperature, some contaminants become more toxic at elevated temperatures and increase the risk of toxic interactions in a global warming context (Hooper et al., 2013). On the other hand, other contaminants become less toxic or remain equally toxic (Weston et al., 2009).

The complex nature of the interactions between toxic substances and temperature has prompted several authors to investigate the issue of combined stresses to improve risk assessment in aquatic environments. Unfortunately, there is a lack of information in the literature on this topic, especially about the effects of chronic low-dose chemical exposure combined to other stresses. In fact, several authors have analysed the effects of xenobiotics, and others have reported the effects of their combination with temperature on fish physiology 
(Hallare et al., 2005; Reynaud and Deschaux, 2006; Li et al., 2014; Andersen et al., 2015; Authman et al., 2015; Guardiola et al., 2015; Olsvik et al., 2016). However, most studies have tested short-term exposure at sub-lethal concentrations which do not reflect actual environmental concentrations of toxicants or the real effects of complex interactions with other stressors such as temperature. Furthermore, health effects may be missed in short-term exposure because adverse impacts can take a long time to emerge (Hamilton et al., 2015).

The present study addresses that issue. We determined the joint effects of chronic environmental exposure to temperature (physical stress) and cadmium (chemical stress) on a small teleost fish often used as an animal model in ecotoxicology: the threespine stickleback (Gasterosteus aculeatus) ( Wootton, 1984; Sanchez et al., 2007).

We selected cadmium as a chemical contaminant model because it is considered as one of the most dangerous inorganic compounds owing to its persistent nature and slow elimination from environmental compartments. This element comes from natural sources and anthropogenic activities such as disposal of industrial, mining, or agricultural wastewater discharges (Sfakianakis et al., 2015). Although the cadmium concentration in fresh aquatic compartments is usually less than $1 \mu \mathrm{g} . \mathrm{L}^{-1}$, it can reach $400 \mu \mathrm{g} . \mathrm{L}^{-1}$ or more in the most contaminated sites (Foran et al., 2002). That is why it has been classified as a priority pollutant in Europe, in line with the EU Water Framework Directive (WFD). Furthermore, information and recommendations on water quality criteria for cadmium are regularly updated in the US Environmental Protection Agency database (US EPA, 2016). Metabolic alterations related to cadmium exposure in fish species are well documented (Soengas et al., 1996; Almeida et al., 2001; Ferrari et al., 2011; Pretto et al., 2014; Pi et al., 2016).

The first purpose of the present study was to evaluate the effects of single or combined exposure to cadmium/temperature stresses on physiological parameters of the threespine stickleback (Gasterosteus aculeatus). Sticklebacks were exposed to an environmental concentration of cadmium (a nominal concentration of $1 \mu \mathrm{g} . \mathrm{L}^{-1}$ ) in combination with two different water temperatures $\left(16{ }^{\circ} \mathrm{C}\right.$ and $\left.21{ }^{\circ} \mathrm{C}\right)$ for three months. We assessed several parameters throughout the experiment: (i) energy metabolism parameters (glycogen, lipid, protein energy reserves, and trypsin, amylase, intestinal alkaline phosphatase enzyme activities), and (ii) antioxidant parameters (superoxide dismutase activity and total glutathione levels). The second purpose was to determine the interest of the three digestive enzymes as biomarkers in comparison with other parameters considered as classical biomarkers in ecotoxicology. 


\section{Materials and methods}

\subsection{Ethics Statement}

The experiment was conducted in accordance with the European directive 2010/63/UE for the protection of animals used for scientific purposes. The registration number of the INERIS, where the experiments were conducted, is C60-769-02. The experimental protocols were submitted and reviewed by an ethical committee nationally recognized in France (CREMEAPS, registration number 96).

\subsection{Fish maintenance conditions}

We used juvenile sticklebacks $(\mathrm{n}=300)$ of $3-3.5 \mathrm{~cm}$ standard length, originating from the same population, and born the same year in the INERIS artificial rivers (Verneuil-enHalatte, France). Three months prior to the experiment, they were transferred from mesocosms to 300-liter laboratory tanks, with a continuous freshwater (0 ppt) circulation system. Water temperature was set at $16{ }^{\circ} \mathrm{C}$, the optimal temperature for sticklebacks reported by Guderley (1994), that was used as a control temperature. Sticklebacks were kept in a thermoregulated room, with a fixed $12 \mathrm{~h} / 12 \mathrm{~h}$ light/dark cycle, and fed on a constant feeding regime (frozen commercial chironomid larvae, $3 \%$ of the fish body weight/day, Europrix, France, ad libitum). The feeding level was adjusted at each time-point and classification, and was maintained as a constant ratio of the fish mass, as described by Leloutre et al. (2016).

\subsection{Experimental design}


Ten days before the onset of the experiment, sticklebacks were randomly distributed in four 90-L laboratory glass tanks (75 per tank) as follows: one control group, and three experimental groups ("cadmium" condition, " $21{ }^{\circ} \mathrm{C}$ " temperature condition, and "cadmiumtemperature" condition) (Figure 1). All along the experiment, sticklebacks were kept in a semistatic freshwater (0 ppt) system and fed similarly to maintenance conditions. Each tank was continuously oxygenated and equipped with a multistage water filter (EHEIM Pro III 2073, Germany) and a water conditioner (TANK ${ }^{\circledR}$ TR20, TECO SRL, Italy). The water temperature of the "cadmium-temperature" and "temperature" tanks was gradually increased at a rate of 0.8 ${ }^{\circ} \mathrm{C}$.day ${ }^{-1}$, from $16{ }^{\circ} \mathrm{C}$ (maintenance temperature) to $21{ }^{\circ} \mathrm{C}$ (experimental temperature), while the “control" and "cadmium" tanks were maintained at $16^{\circ} \mathrm{C}$. We used $21^{\circ} \mathrm{C}$ as a high temperature because it lies within the range of stressful temperatures for sticklebacks (Jordan and Garside, 1972), and slightly exceeds the rise of $1.8-4.0^{\circ} \mathrm{C}$ predicted by global climate change scenarios (IPCC, 2013).

Once the targeted temperature was reached, sticklebacks were kept in the different tanks for 90 days. For the "cadmium" and "cadmium-temperature" conditions, they were exposed to a nominal concentration of $1 \mu \mathrm{g}$. $\mathrm{L}^{-1}$ of cadmium chloride $\left(\mathrm{CdCl}_{2}\right.$, Sigma-Aldrich Chemicals, France). The cadmium concentration was selected in accordance with concentration levels reported in French rivers: 1-10 $\mu \mathrm{g} . \mathrm{L}^{-1}$ (IRSN, 2004). It was four times as much as the European standard reported in the WFD for class 5 inland surface waters (AA-EQS $=0.25 \mu \mathrm{g} . \mathrm{L}^{-1}$ ), and slightly exceeded $0.72 \mu \mathrm{g} . \mathrm{L}^{-1}$, the freshwater chronic criterion (CCC) recommended by the US Environmental Protection Agency (US EPA, 2016).

Throughout the experiment, water was renewed every other day, and water parameters were assessed before and after water replacement. Since water parameters (temperature, $\mathrm{pH}$, conductivity, and dissolved oxygen) remained unchanged before and after water renewal, we presented these data as global means ( \pm SD) (Table 1). Concerning cadmium, water concentrations were monitored before and after water renewal, daily during the first month of exposure and weekly for the rest of the study: water samples were acidified with nitric acid 2 $\%$, and total cadmium concentrations were checked by electrothermal atomic absorption spectrophotometry (SpectrAA, GTA110, Varian, Palo Alto, CA, USA). Since the experiment was conducted in semi-static conditions, cadmium concentrations decreased before water replacement. Hence, the actual cadmium concentration to which sticklebacks were exposed was calculated as the time-weighted mean (TW Mean) according to OECD Test Guidelines (OECD, 
2012), and was half the targeted nominal concentration (Table 1). No mortality was observed during the acclimation or exposure periods.

Table 1. Average water parameters and measured cadmium concentrations recorded during the experiment.

\begin{tabular}{|c|c|c|c|c|c|}
\hline \multirow[b]{2}{*}{ Conditions } & \multicolumn{4}{|c|}{ Water parameters } & \multirow{2}{*}{$\begin{array}{c}\text { Measured } \\
\text { cadmium } \\
\text { concentrations } \\
\left(\mu \mathrm{g} . \mathrm{L}^{-1}\right)\end{array}$} \\
\hline & $\begin{array}{c}\text { Temperature } \\
\left({ }^{\circ} \mathbf{C}\right)\end{array}$ & $\mathbf{p H}$ & $\begin{array}{c}\text { Conductivity } \\
(\mu \mathrm{S})\end{array}$ & $\begin{array}{c}\text { Dissolved Oxygen } \\
(\mathrm{mg} / \mathrm{L})\end{array}$ & \\
\hline Control & $16.10 \pm 0.38$ & $8.33 \pm 0.22$ & $464.60 \pm 64.19$ & $9.40 \pm 0.48$ & $0.01 \pm 0.002$ \\
\hline Cadmium & $16.45 \pm 1.10$ & $8.24 \pm 0.20$ & $484.31 \pm 71.93$ & $9.41 \pm 0.46$ & $0.56 \pm 0.27$ \\
\hline Temperature & $21.13 \pm 0.44$ & $8.14 \pm 0.23$ & $488.46 \pm 59.02$ & $8.51 \pm 0.54$ & $0.01 \pm 0.001$ \\
\hline $\begin{array}{l}\text { Cadmium- } \\
\text { Temperature }\end{array}$ & $21.10 \pm 0.50$ & $8.09 \pm 0.18$ & $524.40 \pm 77.52$ & $8.38 \pm 0.54$ & $0.53 \pm 0.21$ \\
\hline
\end{tabular}

\subsection{Dissection and sample preparation}

Sticklebacks ( $\mathrm{n}=25$ per condition) were sampled on days 15, 60, and 90, after a $24 \mathrm{~h}$ starvation period recommended by Langeland et al. (2013) for digestive enzyme assays. At each sampling date, sticklebacks were anaesthetized with $70 \mathrm{mg}^{-1} \mathrm{~L}^{-1}$ of MS 222, CAS No: 886-862 , and then killed by cervical dislocation (section of the cranial-vertebral junction). Then they were weighed, standard length was measured, and organs (liver, muscle, and the whole digestive tract) were sampled and frozen at $-80{ }^{\circ} \mathrm{C}$, until homogenisation and analysis (Figure $1)$. 
Liver and muscle samples ( $\mathrm{n}=10 /$ condition/sampling date) underwent a common homogenisation step, using ice-cold phosphate buffer (100 mM, pH 7.8) with $20 \%$ glycerol and 0.2 mM PMSF. Liver homogenates were divided into three fractions: A, B, and C. Fraction A was used for cadmium bioaccumulation assays (see paragraph 2.5.), fraction B for antioxidant parameters, and fraction $\mathrm{C}$ for energy reserve parameters (Figure 1).

Fraction $\mathrm{B}$ and muscle homogenates were centrifuged for $15 \min \left(10,000 \mathrm{x} \mathrm{g}, 4^{\circ} \mathrm{C}\right)$, and the supernatants were used to assess antioxidant parameters (Figure 1). Fraction $\mathrm{C}$ was used to assess protein reserves, and then an extraction was performed with chloroform/methanol (1:2; v:v) and sodium sulphate $\left(\mathrm{Na}_{2} \mathrm{SO}_{4}\right) 2 \%$ to assess lipid (in the supernatant) and glycogen (in the pellet), respectively, after a 15 -min centrifugation step $\left(1,000 \mathrm{x} \mathrm{g}, 4{ }^{\circ} \mathrm{C}\right)$.

The whole digestive tracts of all sticklebacks ( $n=25 /$ condition/sampling date) were rinsed on ice using cold Tris- $\mathrm{HCl}$ buffer $(0.01 \mathrm{M}, \mathrm{pH} 7)$, and then homogenised in the same buffer. Digestive homogenates were then centrifuged at $15,000 \mathrm{x} \mathrm{g}\left(30 \mathrm{~min}, 4^{\circ} \mathrm{C}\right)$, and the supernatants were collected and used for enzymatic activity analysis (Figure 1).

Tissue homogenisation (except for carcass) was performed using a PRECELLYS $24^{\circledR}$ homogeniser (BERTIN TECHNOLOGIES, France). 


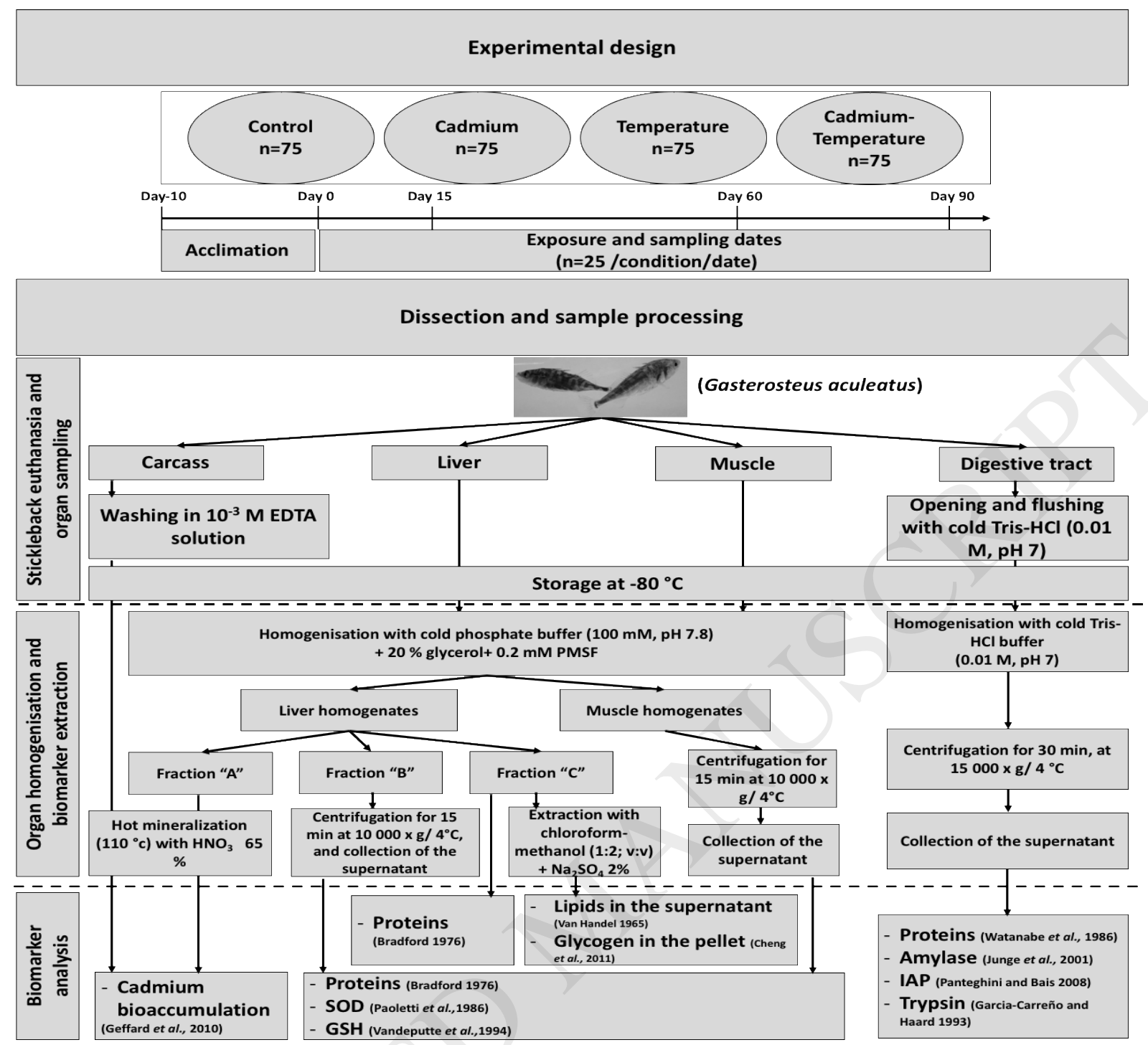

Figure 1. Experimental design and schematic diagram of sample processing and

biomarkers analysis. Fish were sacrificed on days 15, 60 and 90, and organs were sampled to assess cadmium bioaccumulation, antioxidant parameters (superoxide dismutase activity- SOD - and total glutathione levels- GSH -), and energy metabolism parameters: energy reserves (glycogen, lipids, proteins) and digestive enzyme activities (trypsin, amylase, and intestinal alkaline phosphatase - IAP -).

\subsection{Metal analysis}

Cadmium bioaccumulation was assessed in stickleback liver (fraction A) and carcass (Figure 1). Fraction A underwent hot solubilisation with nitric acid at $110{ }^{\circ} \mathrm{C}\left(1 \mathrm{~mL}\right.$ of $\mathrm{HNO}_{3}$ for $1 \mathrm{~mL}$ of homogenate), and samples were diluted with ultrapure water before cadmium analysis. Stickleback carcasses (after the kidney, spleen, and gonads were removed), were washed in a solution of $10^{-3}$ MEDTA to remove cadmium from the fish surface, and frozen at $-80{ }^{\circ} \mathrm{C}$ until mineralisation and cadmium measurements (Figure 1). Carcass tissues were 
digested individually at $110{ }^{\circ} \mathrm{C}$ by adding $5 \mathrm{~mL}$ of concentrated nitric acid $\left(\mathrm{HNO}_{3} 65 \%\right)$. Carcass digestates were then adjusted to $10 \mathrm{~mL}$ with ultrapure $\mathrm{H}_{2} \mathrm{O}$ before cadmium analysis. After the mineralisation step, cadmium was assayed in all samples by electrothermal atomic absorption spectrophotometry (SpectrAA, GTA110, Varian, Palo Alto, CA, USA), as described by Geffard et al. (2010). Results were expressed as $\mu \mathrm{g}$. $\mathrm{g}^{-1}$ wet wt.

\subsection{Biological analyses}

\subsubsection{Fish biometric parameters}

Stickleback growth was assessed by monitoring the evolution of their weight and length throughout the experiment. Sex was determined, and the gonado-somatic index (GSI = gonad mass $(\mathrm{g}) \times$ [body mass $\left.(\mathrm{g})]^{-1} \times 100\right)$ and Fulton's condition factor $(\mathrm{K}=$ Weight $(\mathrm{g}) \times[$ Length $\left.\left.(\mathrm{mm})^{3}\right]^{-1} \times 100\right)$ were measured.

\subsubsection{Oxidative stress parameters}

Before GSH and SOD activity assays, cytosolic protein concentrations were determined according to Bradford's (1976) method using bovine serum albumin (Sigma-Aldrich Chemicals, France) as a standard. Antioxidant biomarkers were assayed according to the methods of Vandeputte et al. (1994) and Paoletti et al. (1986), respectively, adapted to microplates and optimised for threespine stickleback as described by Sanchez et al. (2007). Results were expressed as $\mathrm{U} . \mathrm{g}^{-1}$ of protein for SOD activity and $\mu \mathrm{mol} . \mathrm{g}^{-1}$ of protein for GSH.

\subsubsection{Energy metabolism parameters}

\section{a) Energy reserves}

Protein contents were measured according to Bradford's (1976) method using bovine serum albumin (Sigma-Aldrich Chemicals, France) as a standard. Lipid and glycogen were measured according to protocols adapted from Van Handel (1965) and Cheng et al. (2011), respectively, using triolein (Sigma-Aldrich Chemicals, France) and glucose solutions (SigmaAldrich Chemicals, France) as standards.

\section{b) Digestive enzymes activities}

Amylase, intestinal alkaline phosphatase (IAP) activities and cytosolic gut protein contents were measured according to the methods of Junge et al. (2001), Panteghini and Bais (2008), and Watanabe et al. (1986), respectively, using Thermo-Scientific Gallery ready-to-use 
reagents. Trypsin activity was measured according to Garcia-Carreño and Haard (1993), using N-benzoyl-DL-arginine 4-nitroanilide hydrochloride (BAPNA, $3 \mathrm{mM}$ ) as a substrate. All enzymatic techniques were conducted at $37{ }^{\circ} \mathrm{C}$ by kinetic colorimetric assay at $405 \mathrm{~nm}$, adapted on an automated photometric analyser (Gallery ${ }^{\mathrm{TM}}$, Thermo Fisher Scientific Oy) and optimised for threespine stickleback. Each enzyme activity was expressed as U. $\mathrm{g}^{-1}$ of protein.

\subsection{Statistical analyses}

Statistical analyses were performed using R statistical software (v3.3.1) with $\alpha=0.05$. Before statistical treatment, normality and homogeneity tests (Shapiro's and Levene's tests, respectively) were performed, followed by a parametric test (two-factor ANOVA), with time point and treatment group as factors. When data were not normally distributed, log, Box-Cox or square-root transformation were used. Tukey's HSD post-hoc test was performed to compare conditions at each time-point. Results were expressed as means \pm SEs.

\section{Results}

\subsection{Cadmium bioaccumulation}

We assessed cadmium bioaccumulation in G. aculeatus carcass (Figure 2 A) and liver (Figure 2 B) after 15, 60, and 90 days of exposure. No bioaccumulation was observed in carcass whatever the experimental condition (Figure 2 A). However, in liver, cadmium bioaccumulated in both "cadmium" and "cadmium-temperature" conditions. Although cadmium concentrations did not significantly differ between the two groups at each sampling date, the trends of the bioaccumulation kinetics differed, but nonsignificantly (Figure 2 B).

Compared to the initial values of day 15 , cadmium bioaccumulation was significantly higher (1.5- to 1.9-fold) on days 60 and 90 in the "cadmium" and "cadmium-temperature" groups ( $\mathrm{p}<$ 0.01 ), whereas no bioaccumulation was observed in the control and "temperature" groups. 


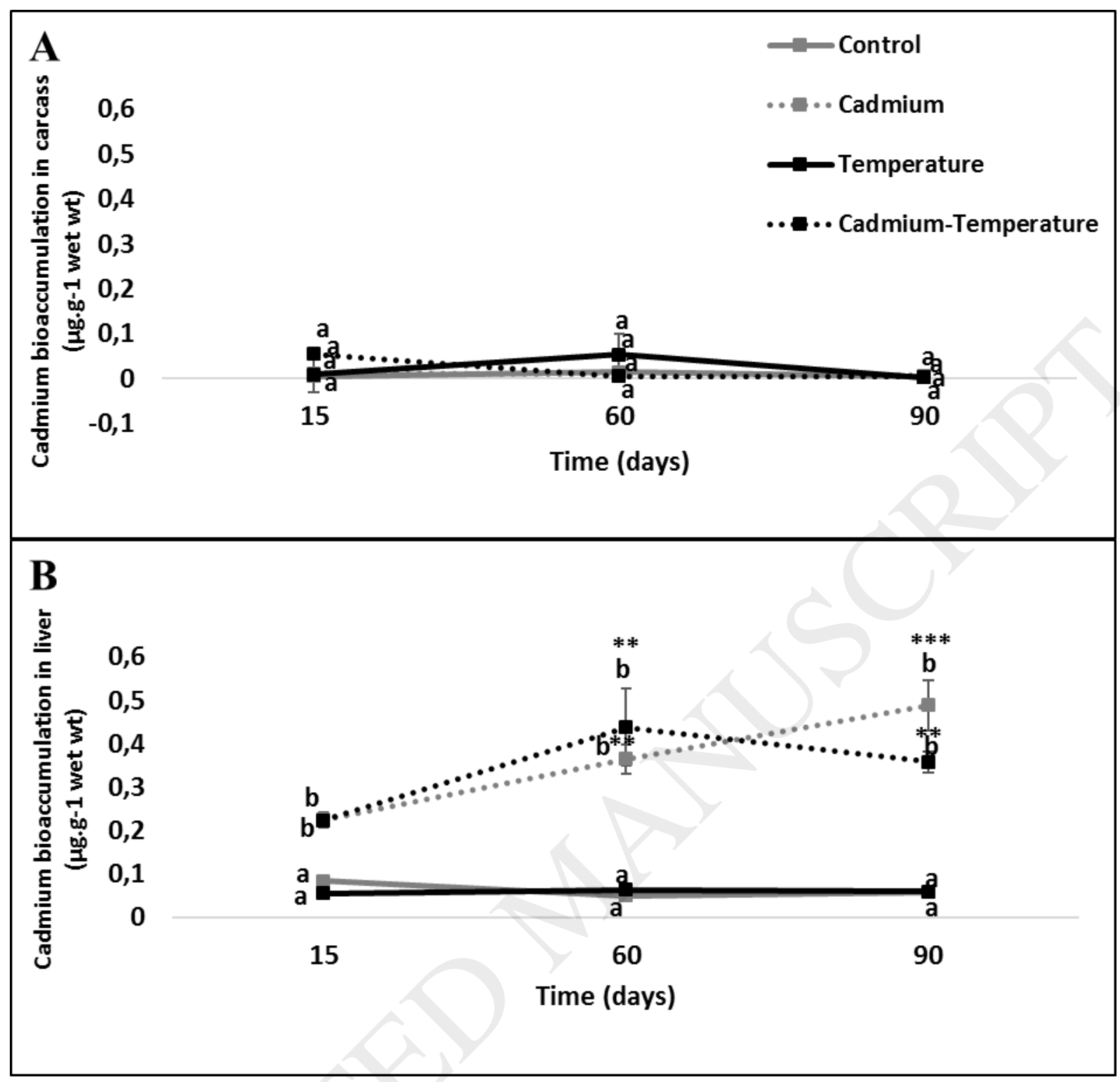

Figure 2. Cadmium bioaccumulation in G. aculeatus carcass (A) and liver (B) after 15, 60, and 90 days. Sticklebacks nonexposed to cadmium and kept at $16^{\circ} \mathrm{C}$ were considered as

the control group. For a same time point, different letters indicate significant differences between groups (mean \pm S.E; $\mathrm{n}=10, \mathrm{p}<0.05$ ). For a same group, asterisks indicate significant temporal differences with respect to initial values on day 15 $(* \mathrm{p}<0.05 ; * * \mathrm{p}<0.01 ; * * * \mathrm{p}<0.001)$.

\subsection{Stickleback growth and biometric parameters}

As regards weight and length at any given time point, no difference was noted between the treated and control groups $(\mathrm{p}<0.05)$, except on day 90, when weight $(1.3$-fold, $\mathrm{p}<0.01)$ and length $(1.15$-fold, $\mathrm{p}<0.01)$ significantly decreased in sticklebacks from the "temperature" and "cadmium-temperature" groups as compared to the control group. However, a significant 
temporal increase in weight and length was noted in all groups on days 60 and 90 as compared to initial values of day 15 (Table 2).

As regards Fulton's condition factor at any given time point, no significant difference was noted between the treated and control groups, except on day 90 when Fulton's condition factor was significantly higher in the treated groups than in the control group (1.2-fold, $\mathrm{p}<0.01)$. Whatever the condition, no temporal effect was observed on Fulton's index when compared to the initial values of day 15 , except a significant 1.27 -fold decrease on day $90(\mathrm{p}=0.0001)$ in the control group.

As for the GSI, no significant difference was observed between exposed and control groups in males. Whatever the condition, no temporal effect was observed, except in males of the cadmium group, in which a significant 1.8-fold decrease was recorded on days 60 and 90 as compared to the initial value of day 15 ( $\mathrm{p}<0.05)$. In females, GSI values were negatively impacted by the elevated temperature and its combination with cadmium on day 90 as compared to the control and "cadmium" groups $(\mathrm{p}<0.05)$. A temporal effect was observed in all female groups when compared to initial values of day 15. In fact, in the control and "cadmium" conditions, GSI values increased significantly from day 60 , to reach the highest values on day 90 (5-fold higher than values on day $15, \mathrm{p}<0.0001$ ). However, in female sticklebacks from the "temperature" and "cadmium-temperature" groups, the highest GSI values were observed on day 60 (5.7- to 6-fold higher than on day 15, p<0.001), followed by a sharp decrease on day 90 to reach similar values to those of day 15 . 
Table 2. Evolution of fish biometric parameters, anti-oxidant parameters, and energy reserves under the effect of temperature and/or cadmium exposure, after 15, 60, and 90 days. For a same time point, different letters indicate significant differences between exposed groups (mean \pm S.E; $n=10$, $p<0.05)$. For a same exposed group, asterisks indicate significant temporal differences as compared to initial values of day $15\left({ }^{*} \mathrm{p}<0.05 ; * * \mathrm{p}<0.01 ; * * * \mathrm{p}<0.001\right)$.

\begin{tabular}{|c|c|c|c|c|c|c|c|c|c|c|c|c|c|}
\hline & \multirow{3}{*}{ Conditions } & \multicolumn{5}{|c|}{ Fish biometric parameters } & \multicolumn{4}{|c|}{ Oxidative stress } & \multicolumn{3}{|c|}{ Energy reserves (J) } \\
\hline & & \multirow{2}{*}{ Weight (mg) } & \multirow{2}{*}{ Length (mm) } & \multirow{2}{*}{$\begin{array}{c}\text { Fulton's } \\
\text { factor }(\mathbf{K})\end{array}$} & \multicolumn{2}{|r|}{ GSI } & \multirow{2}{*}{$\begin{array}{c}\text { Liver } \\
\text { GSH } \\
\left(\mu \text { mol. } \mathbf{g}^{-1}\right. \\
\text { of prot })\end{array}$} & \multirow{2}{*}{$\begin{array}{c}\text { Muscle } \\
\text { GSH } \\
\left(\mu \mathrm{mol} . \mathrm{g}^{-1}\right. \\
\text { of prot })\end{array}$} & \multirow{2}{*}{$\begin{array}{c}\text { Liver } \\
\text { SOD } \\
\left.\text { (U. } \mathbf{g}^{-1} \text { of prot }\right)\end{array}$} & \multirow{2}{*}{$\begin{array}{c}\text { Muscle } \\
\text { SOD } \\
\text { (U. } \mathbf{g}^{-1} \text { of prot) }\end{array}$} & \multirow{2}{*}{ Glycogen } & \multirow{2}{*}{ Lipids } & \multirow{2}{*}{ Proteins } \\
\hline & & & & & Males & Females & & & & & & & \\
\hline \multirow{4}{*}{$\begin{array}{c}\text { Day } \\
15\end{array}$} & Control & $\begin{array}{l}618.60 \pm \\
51.83 \mathbf{a}\end{array}$ & $35.20 \pm 0.91 \mathbf{a}$ & $\begin{array}{r}1.38 \pm \\
0.02 \mathbf{a}\end{array}$ & $\begin{array}{r}1.34 \pm \\
0.49 \mathbf{a b}\end{array}$ & $2.31 \pm 1.74 \mathrm{a}$ & $\begin{array}{l}9.77 \pm \\
0.59 \mathbf{a}\end{array}$ & $4.66 \pm 0.39 \mathbf{a}$ & $1304.78 \pm 98.71 \mathbf{a}$ & $\begin{array}{c}479.43 \pm \\
54.53 \mathbf{a}\end{array}$ & $11.59 \pm 2.03 \mathbf{a}$ & $\begin{array}{l}88.56 \pm \\
13.89 \mathbf{a}\end{array}$ & $\begin{array}{r}95.58 \pm \\
11.71 \mathbf{a}\end{array}$ \\
\hline & Cadmium & $\begin{array}{l}659.50 \pm \\
43.95 \mathbf{a}\end{array}$ & $36.9 \pm 0.69 \mathbf{a}$ & $\begin{array}{l}1.29 \pm \\
0.04 a\end{array}$ & $\begin{array}{l}1.79 \pm \\
0.70 \mathbf{a}\end{array}$ & $2.22 \pm 0.96 \mathbf{a}$ & $\begin{array}{l}8.47 \pm \\
0.66 \mathbf{a}\end{array}$ & $4.07 \pm 0.49 \mathbf{a}$ & $1726.37 \pm 116.33 \mathbf{a b}$ & $\begin{array}{l}524.82 \pm \\
131.04 \mathbf{a}\end{array}$ & $19.18 \pm 2.66 \mathbf{a}$ & $\begin{array}{c}104.06 \pm \\
13.37 \mathbf{a}\end{array}$ & $\begin{array}{c}118.09 \pm \\
12.31 \mathrm{a}\end{array}$ \\
\hline & Temperature & $\begin{array}{c}573.20 \pm \\
56.59 \mathrm{a}\end{array}$ & $34.5 \pm 1.18 \mathrm{a}$ & $\begin{array}{r}1.36 \pm \\
0.03 \mathbf{a}\end{array}$ & $\begin{array}{l}0.83 \pm \\
0.74 \mathbf{b}\end{array}$ & $1.80 \pm 0.57 \mathbf{a}$ & $\begin{array}{r}7.98 \pm \\
0.61 \mathbf{a}\end{array}$ & $4.52 \pm 0.30 \mathbf{a}$ & $1666.71 \pm 176.46 \mathbf{a b}$ & $\begin{array}{c}601.66 \pm \\
34.41 \mathrm{a}\end{array}$ & $16.18 \pm 1.83 \mathbf{a}$ & $\begin{array}{c}102.25 \pm \\
8.49 \mathrm{a}\end{array}$ & $\begin{array}{c}92.65 \pm \\
10.07 \mathbf{a}\end{array}$ \\
\hline & $\begin{array}{c}\text { Cadmium- } \\
\text { Temperature }\end{array}$ & $\begin{array}{c}535.40 \pm \\
49.36 \mathbf{a}\end{array}$ & $34.10 \pm 1.12 \mathbf{a}$ & $\begin{array}{r}1.31 \pm \\
0.02 \mathbf{a}\end{array}$ & $\begin{array}{l}0.87 \pm \\
0.54 \mathbf{b}\end{array}$ & $1.92 \pm 0.86 \mathbf{a}$ & $\begin{array}{l}8.54 \pm \\
1.39 \mathbf{a}\end{array}$ & $4.19 \pm 0.46 \mathbf{a}$ & $1987.34 \pm 56.60 \mathbf{b}$ & $\begin{array}{c}566.13 \pm \\
63.05 \mathbf{a}\end{array}$ & $12.61 \pm 2.43 \mathbf{a}$ & $\begin{array}{l}86.82 \pm \\
10.97 \mathbf{a}\end{array}$ & $\begin{array}{l}83.14 \pm \\
10.13 \mathbf{a}\end{array}$ \\
\hline \multirow{4}{*}{$\begin{array}{c}\text { Day } \\
60\end{array}$} & Control & $\begin{array}{l}1015.10 \pm \\
89.55 \mathbf{a}^{* * *}\end{array}$ & $\begin{array}{l}41.30 \pm \\
0.90 \mathbf{a}^{*}\end{array}$ & $\begin{array}{l}1.40 \pm \\
0.03 \mathbf{a}\end{array}$ & $\begin{array}{l}1.16 \pm \\
0.50 \mathbf{a}\end{array}$ & $7.35 \pm 4.72 \mathbf{a}^{* * * *}$ & $\begin{array}{r}9.94 \pm \\
0.99 \mathbf{a}\end{array}$ & $3.81 \pm 0.31 \mathbf{a}$ & $1622.01 \pm 201.77 \mathbf{a}$ & $\begin{array}{l}886.22 \pm \\
170.65 \mathbf{a}\end{array}$ & $40.24 \pm 7.97 \mathbf{a}^{* * *}$ & $\begin{array}{l}160.88 \pm \\
14.98 \mathbf{a}^{* * *}\end{array}$ & $\begin{array}{l}197.07 \pm \\
20.60 \mathbf{a}^{*}\end{array}$ \\
\hline & Cadmium & $\begin{array}{l}1098.8 \pm \\
71.57 \mathbf{a}^{* * *}\end{array}$ & $\begin{array}{l}42.40 \pm \\
0.76 \mathbf{a}^{* *}\end{array}$ & $\begin{array}{l}1.42 \pm \\
0.03 \mathbf{a}\end{array}$ & $\begin{array}{c}0.97 \pm \\
0.34 \mathbf{a}^{* *}\end{array}$ & $9.79 \pm 6.19 \mathbf{a}^{\text {*****}}$ & $\begin{array}{r}9.98 \pm \\
0.84 a\end{array}$ & $\begin{array}{l}4.08 \pm \\
0.39 \mathbf{a c}\end{array}$ & $1788.74 \pm 204.30 \mathbf{a}$ & $\begin{array}{l}818.56 \pm \\
215.89 \mathbf{a}\end{array}$ & $53.42 \pm 8.08 \mathbf{a}^{* * * *}$ & $\begin{array}{l}182.86 \pm \\
15.41 \mathbf{a}^{* *}\end{array}$ & $\begin{array}{l}263.56 \pm \\
24.16 \mathbf{b}^{* * *}\end{array}$ \\
\hline & Temperature & $\begin{array}{r}1009.5 \pm \\
51.39 \mathbf{a}^{* * * * *}\end{array}$ & $\begin{array}{c}40.80 \pm \\
0.46 \mathbf{a}^{* * * * *}\end{array}$ & $\begin{array}{l}1.47 \pm \\
0.03 \mathbf{a}\end{array}$ & $\begin{array}{c}0.87 \pm \\
0.45 \mathbf{a}\end{array}$ & $11.89 \pm 7.36 \mathbf{a}^{* * *}$ & $\begin{array}{c}10.64 \pm \\
1.26 \mathbf{a}\end{array}$ & $5.88 \pm 0.55 \mathbf{b}$ & $2039.11 \pm 208.22 \mathbf{a}$ & $\begin{array}{l}1062.84 \pm \\
148.67 \mathbf{a}^{*}\end{array}$ & $47.27 \pm 8.15 \mathbf{a}^{* *}$ & $\begin{array}{l}147.06 \pm \\
10.78 \mathbf{a}^{*}\end{array}$ & $\begin{array}{r}195.40 \pm \\
11.30 \mathbf{a}^{* * * * *}\end{array}$ \\
\hline & $\begin{array}{l}\text { Cadmium- } \\
\text { Temperature }\end{array}$ & $\begin{array}{c}939.00 \pm \\
47.72 \mathbf{a}^{* * * *}\end{array}$ & $\begin{array}{l}40.90 \pm \\
0.88 \mathbf{a}^{* * * *}\end{array}$ & $\begin{array}{l}1.37 \pm \\
0.03 \mathbf{a}\end{array}$ & $\begin{array}{c}0.99 \pm \\
0.57 \mathbf{a}\end{array}$ & $11.07 \pm 5.13 \mathbf{a}^{* * *}$ & $\begin{array}{c}10.95 \pm \\
1.17 \mathbf{a}\end{array}$ & $\begin{array}{l}5.15 \pm \\
0.53 \mathbf{b c}\end{array}$ & $1850.28 \pm 108.17 \mathbf{a}$ & $\begin{array}{r}944.58 \pm \\
199.60 \mathbf{a}\end{array}$ & $28.59 \pm 4.39 \mathbf{a}^{*}$ & $\begin{array}{c}163.62 \pm \\
11.29 \mathbf{a}^{* * * * *}\end{array}$ & $\begin{array}{r}204.03 \pm \\
15.19 \mathbf{a b}^{* * * *}\end{array}$ \\
\hline \multirow{4}{*}{$\begin{array}{c}\text { Day } \\
\mathbf{9 0}\end{array}$} & Control & $\begin{array}{c}1134.80 \pm \\
111.02 \mathbf{a}^{* * * * * *}\end{array}$ & $\begin{array}{c}47.10 \pm \\
2.19 \mathbf{a}^{* * * *}\end{array}$ & $\begin{array}{c}1.08 \pm \\
0.06 \mathbf{a}^{* * * *}\end{array}$ & $\begin{array}{l}0.90 \pm \\
0.50 \mathbf{a}\end{array}$ & $12.36 \pm 7.12 \mathbf{a}^{* * *}$ & $\begin{array}{l}5.45 \pm \\
0.63 \mathrm{a}^{*}\end{array}$ & $4.55 \pm 0.36 \mathbf{a}$ & $737.76 \pm 83.88 \mathbf{a}$ & $279.98 \pm 9.73 \mathbf{a}$ & $25.62 \pm 2.18 \mathbf{a}^{* *}$ & $\begin{array}{c}206.55 \pm \\
17.64 \mathbf{a}^{* * * *}\end{array}$ & $\begin{array}{c}291.10 \pm \\
40.12 \mathbf{a}^{* * * *}\end{array}$ \\
\hline & Cadmium & $\begin{array}{l}1123.50 \pm \\
115.01 \mathbf{a}^{* * *}\end{array}$ & $\begin{array}{l}43.10 \pm \\
1.44 \mathbf{a}^{* * * *}\end{array}$ & $\begin{array}{l}1.36 \pm \\
0.03 \mathbf{b}\end{array}$ & $\begin{array}{l}0.92 \pm \\
0.47 \mathbf{a}^{*}\end{array}$ & $12.94 \pm 7.09 \mathbf{a}^{* * * *}$ & $\begin{array}{l}6.49 \pm \\
0.83 \mathbf{a}\end{array}$ & $4.36 \pm 0.45 \mathbf{a}$ & $1054.15 \pm 170.73 \mathbf{a b}$ & $\begin{array}{c}289.64 \pm \\
12.55 \mathbf{a}\end{array}$ & $29.66 \pm 5.31 \mathbf{a}$ & $\begin{array}{c}224.38 \pm \\
21.71 \mathbf{a}^{* * * * *}\end{array}$ & $\begin{array}{l}290.38 \pm \\
42.77 \mathbf{a}^{* * * * * *}\end{array}$ \\
\hline & Temperature & $\begin{array}{l}873.9 \pm \\
51.71 \mathbf{b}^{* * *}\end{array}$ & $\begin{array}{c}40.80 \pm \\
1,37 \mathbf{b}^{* * * * *}\end{array}$ & $\begin{array}{l}1.29 \pm \\
0.06 \mathbf{b}\end{array}$ & $\begin{array}{c}0.40 \pm \\
0.29 \mathbf{a}\end{array}$ & $2.05 \pm 1.48 \mathbf{b}$ & $\begin{array}{l}5.18 \pm \\
0.83 \mathbf{a}\end{array}$ & $5.78 \pm 0.41 \mathbf{a}$ & $990.07 \pm 100.65 \mathbf{a b}^{*}$ & $\begin{array}{l}570.77 \pm \\
61.26 \mathbf{b}\end{array}$ & $25.84 \pm 5.64 \mathbf{a}$ & $\begin{array}{c}168.25 \pm \\
10.62 \mathbf{b}^{* * * *}\end{array}$ & $\begin{array}{l}172.49 \pm \\
23.67 \mathbf{b}^{* * *}\end{array}$ \\
\hline & $\begin{array}{l}\text { Cadmium- } \\
\text { Temperature }\end{array}$ & $\begin{array}{l}813.00 \pm \\
56.81 b^{* * *}\end{array}$ & $\begin{array}{l}39.30 \pm \\
1.18 \mathbf{b}^{* *}\end{array}$ & $\begin{array}{l}1.32 \pm \\
0.03 \mathbf{b}\end{array}$ & $\begin{array}{c}0.54 \pm \\
0.29 \mathbf{a}\end{array}$ & $4.63 \pm 4.41 \mathbf{b}$ & $\begin{array}{l}8.23 \pm \\
0.28 \mathbf{a}\end{array}$ & $4.87 \pm 0.42 \mathbf{a}$ & $\begin{array}{l}1401.87 \pm \\
102.62 b^{* * *}\end{array}$ & $\begin{array}{l}483.32 \pm \\
65.38 \mathbf{b}\end{array}$ & $36.91 \pm 5.83 \mathbf{a}^{* *}$ & $\begin{array}{c}168.41 \pm \\
10.45 \mathbf{b}^{* * * *}\end{array}$ & $\begin{aligned} 172.76 \pm \\
19.56 \mathbf{b}^{* * * * *}\end{aligned}$ \\
\hline
\end{tabular}




\subsection{Oxidative stress parameters}

There was no significant difference in GSH activity (Table 2) between treated groups all along the experiment, except in muscle, in which a significant 1.3-fold increase was noted on day $60(p<0.05)$ in the sticklebacks of the "temperature" and "cadmium-temperature" conditions as compared to the control group. Furthermore, no temporal effect was observed on liver or muscle GSH levels in any group.

As regards SOD activity, a consistent trend was recorded in both liver and muscle all along the experiment. In fact, SOD activity was higher in the treated groups than in the control groups. But the difference was significant in liver only on days 15 ( 1.5 -fold, $\mathrm{p}=0.04)$ and 90 (1.9-fold, $\mathrm{p}=0.004)$ in the "cadmium-temperature" group, and in muscle only on day 90 (1.7fold, $\mathrm{p}=0.01$ ), in the "temperature" and "cadmium-temperature" groups. A temporal effect was observed only on day 90 in liver and on day 60 in muscle. In liver, a significant decrease of SOD activity was observed in the "temperature" (1.6-fold, $\mathrm{p}=0.04)$ and "cadmium-temperature" (1.4-fold, $\mathrm{p}=0.006$ ) groups as compared to initial values of day 15 . However, in muscle, a significant increase of SOD activity occurred in the "temperature" group (1.7-fold, p=0.04) only on day 60 as compared to initial values of day 15 .

\subsection{Energy metabolism parameters}

\subsubsection{Energy reserves}

For a same date, neither cadmium nor temperature, nor again their combination had any significant effect on glycogen throughout the experiment (Table 2). However, when compared to initial values of day 15 , a significant 2- to 3 -fold increase in glycogen values was observed in all groups on day $60(\mathrm{p}<0.05)$, but only in the control and "cadmium-temperature" groups on day 90 (2-fold increase, $\mathrm{p}<0.01$ ).

Lipid and protein quantities exhibited the same patterns throughout the experiment. In fact, no significant difference was noted between the treated groups on days 15 and 60 (p>0.05). However, as compared to the control and "cadmium" groups, a significant decrease of protein and lipid quantities was recorded on day 90 (1.6-fold, $\mathrm{p}=0.009)$ in the "temperature" and "cadmium-temperature" groups. A significant temporal increase of lipid and protein quantities was observed all along the experiment in all groups $(1.5$ to 3 -fold increase, $p<0.05)$. 


\subsubsection{Digestive enzyme activities}

Throughout the experiment, IAP and trypsin activities were highly modulated in response to cadmium, temperature, and their combination, and followed a similar trend in all groups (Figures $3 \mathrm{~A}$ and $3 \mathrm{~B}$ ). In the control groups, when compared to initial values of day 15, a significant increase of IAP activity was noted on day $90(\mathrm{p}<0.0001)$, whereas a significant decrease of trypsin activity $(\mathrm{p}=0.006)$ was observed on day 60 , followed by a significant increase $(\mathrm{p}<0.0001)$ on day 90. Compared to the control groups, cadmium had a significant effect only on day 90, and was responsible for ca. 2.9-fold and 1.9-fold reductions of IAP and trypsin activities, respectively $(\mathrm{p}<0.0001)$. Temperature caused a significant decrease in IAP and trypsin activities from day 15 until day 90 (7-fold decrease, $\mathrm{p}<0.0001$ ). The effect of cadmium on the activity of the two digestive enzymes was aggravated in the presence of temperature stress, and was observed earlier after day 15. In fact, IAP and trypsin activity levels significantly decreased by 2.5 to 4.5 -fold on days 15 and 60 ( $\mathrm{p}<0.00001)$, and 7-fold on day 90 $(\mathrm{p}<0.00001)$ in the "cadmium-temperature" groups as compared to the control groups.

Amylase activity (Figure $3 \mathrm{C}$ ) changed less than trypsin and IAP activities when sticklebacks were exposed to cadmium or temperature, with a significant 1.3-fold decrease from day $60(\mathrm{p}<0.05)$ in the "temperature" group, and 2.1-fold only on day $90(\mathrm{p}=0.01)$ in the "cadmium" group. Exposure to the higher temperature enhanced the effects of cadmium on amylase activity: in the "cadmium-temperature" groups as compared to the control groups, amylase activity decreased significantly earlier, similarly to IAP and trypsin activities, from day 15 (1.5-fold decrease, $\mathrm{p}<0.001)$, and remained reduced until day 90 (2.1-fold decrease, $\mathrm{p}=0.01$ ). Compared to initial values on day 15 , amylase activity remained unchanged in all groups throughout the experiment, except in the control group on day 60 (1.4-fold increase, $\mathrm{p}<0.01)$ and in the cadmium group on day 90 (1.3-fold decrease, $\mathrm{p}<0.05)$. 


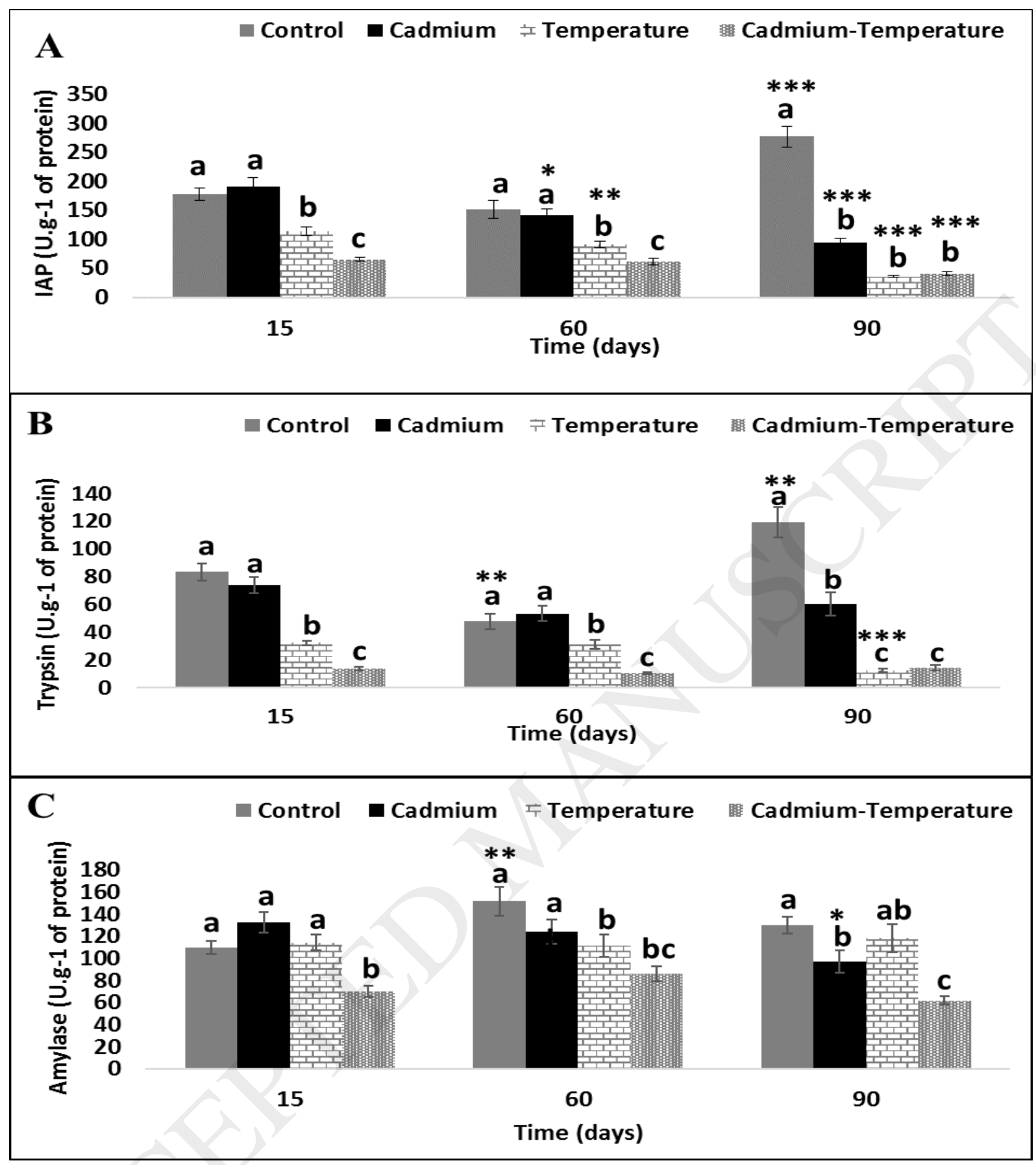

Figure 3. Intestinal alkaline phosphatase (IAP) (A), trypsin (B) and amylase (C) activities under the effect of temperature and/or cadmium after 15, 60, and 90 days. Sticklebacks maintained at $16^{\circ} \mathrm{C}$ and non-exposed to cadmium were considered as the control group.

For a same time point, different letters indicate significant differences between exposed groups (mean \pm S.E; $n=25, p<0.05$ ). For a same exposed group, asterisks indicate significant temporal differences with respect to initial values of day $15\left({ }^{*} \mathrm{p}<0.05\right.$; $* * \mathrm{p}<0.01 ; * * * \mathrm{p}<0.001)$. 


\section{Discussion}

\subsection{Cadmium exposure and bioaccumulation}

We conducted our experiment in semi-static conditions, and water was renewed every other day to keep the cadmium concentration constant. Although a nominal concentration of 1 $\mu \mathrm{g} . \mathrm{L}^{-1}$ of cadmium was targeted, the real water cadmium concentration calculated at the end of the experiment was 2 -fold lower, i.e. $0.5 \mu \mathrm{g} . \mathrm{L}^{-1}$. Therefore sticklebacks were exposed chronically to a very low cadmium concentration, below the freshwater chronic criterion (CCC) recommended by the US Environmental Protection Agency: $0.72 \mu \mathrm{g} . \mathrm{L}^{-1}$ (US EPA, 2016), and 2-fold higher than the annual average value (AA-EQS $=0.25 \mu \mathrm{g} . \mathrm{L}^{-1}$ ) reported by the European Directive 2013/39/EU for inland surface waters (Class 5). Despite this very low concentration (supported by our bioaccumulation results), we noted adverse effects on certain parameters throughout the experiment. In fact, cadmium accumulated in liver but not in carcass. These results are not surprising considering the very low concentration to which sticklebacks were exposed. The literature reports that even at high concentrations, cadmium accumulates at higher rates in liver than in carcass. Moreover, the liver is the main xenobiotic-detoxifying organ (Cazenave et al., 2006; Vergauwen et al., 2013). Contrary to our expectations, cadmium accumulation in liver was not influenced by the elevated temperature, and no correlation was observed between these two parameters. This goes against literature results, since increased metal accumulation along with increasing temperature has been widely reported in many species as one of the mechanisms whereby temperature influences metal toxicity (Vergauwen et al., 2013; Perera et al., 2015).

\subsection{Effects on biometric parameters}

Temperature is a potent physical stressor for fish, with major effects on the metabolism, growth, and fundamental biochemical processes (Hovel et al., 2015). It had a major effect on stickleback biometric parameters: at $21^{\circ} \mathrm{C}$, sticklebacks (exposed to cadmium or not) weighed less than those maintained at $16{ }^{\circ} \mathrm{C}$ (with or without cadmium exposure) after 90 days of exposure. These results confirm that sticklebacks, as a cold-water species, prefer cool temperatures $\left(<18{ }^{\circ} \mathrm{C}\right)$ (Guderley, 1994). They also suggest that $21{ }^{\circ} \mathrm{C}$ is an upper tolerance limit for their long-term growth, even if they are fed ad libitum, a rare occurrence in the natural habitats of this species (Wootton, 1984). Similarly, the growth capacity of the coral reef fish Acanthochromis polyacanthus was severely limited, even with high food rations, after 3 months of exposure to a high water temperature (Munday et al., 2008).

Concerning Fulton's condition factor, neither temperature nor cadmium had an effect on sticklebacks. However, a decrease was observed in the control fish on day 90 and could be 
explained by the increase of fish length, since the $\mathrm{K}$-factor is a crude parameter that takes into account fish weight and the cube of fish length. The GSI was not impacted by cadmium exposure in females, but was highly influenced by temperature conditions (alone or in combination with cadmium): at $21{ }^{\circ} \mathrm{C}$ (with or without cadmium exposure), the GSI peaked on day 60 , whereas at $16^{\circ} \mathrm{C}$ (with or without cadmium exposure), the peak was reached only after 90 days. These results suggest that oocyte formation was promoted earlier in females raised at $21{ }^{\circ} \mathrm{C}$. High temperature has been reported to augment gonadal development in teleost fish, including sticklebacks (Guderley, 1994; Acharia et al., 2014). However, degeneration of gonad morphology following exposure to a constant high temperature has been reported too (Acharia et al., 2014). This may explain why the GSI sharply decreased on day 90 in sticklebacks raised at $21{ }^{\circ} \mathrm{C}$ (exposed to cadmium or not), suggesting impaired reproduction.

\subsection{Effects on core biomarkers}

\subsubsection{Antioxidant parameters}

Despite a nonsignificant increasing trend of SOD activity in the cadmium-treated groups as compared to the control groups, we detected no sign of oxidative stress. Heavy metals are known to accumulate in fish tissues and catalyse reactions that generate reactive oxygen species (ROS) and lead to oxidative stress. In fish, antioxidant parameters can be induced or inhibited by heavy metals depending on the dose, the species, and the organ (Sanchez et al., 2005; Farombi et al., 2007; Zheng et al., 2016). In our study, the absence of oxidative stress at this concentration could be due to the protective role of other organs (i.e. the gut) that may have sequestered cadmium more than the liver and muscle did, resulting in effects other than those of oxidative stress. Cadmium compartmentalisation and sequestration in the digestive tract has been reported in fish exposed to waterborne heavy metals (Andres et al., 2000; Vergauwen et al., 2013). Unfortunately, we did not study cadmium bioaccumulation in the digestive tract to support this hypothesis.

Although cadmium had no impact on oxidative stress, temperature (alone or in combination with cadmium) influenced the two antioxidant parameters. GSH levels significantly increased in muscle on day 60 , and SOD activity also significantly increased in liver on days 15 and 90, and in muscle only on day 90. Several studies have shown that oxidative stress biomarkers are highly sensitive to temperature, but their response depends on fish species (Figueiredo-Fernandes et al., 2006; Vinagre et al., 2012; Madeira et al., 2013). In Nile tilapia (Oreochromis niloticus) raised at 17 and $27^{\circ} \mathrm{C}$ for 45 days, no difference was observed in SOD or GST activity (Figueiredo-Fernandes et al., 2006). However, in juvenile seabass (Dicentrarchus labrax), catalase activity was higher at $18{ }^{\circ} \mathrm{C}$ and $28{ }^{\circ} \mathrm{C}$ than at $24{ }^{\circ} \mathrm{C}$, their 
thermal optimum. The authors concluded that fish experience thermal stress at temperatures beyond their thermal optimum (Vinagre et al., 2012). Sticklebacks are typically present in waters where temperatures range from 4 to $20^{\circ} \mathrm{C}$ (Wootton, 1984). Even if a temperature of 21 ${ }^{\circ} \mathrm{C}$ slightly exceeds their optimal thermal range, they may be exposed to this temperature in natural habitats now and then. Long-term exposure to this temperature seems to be stressful, and these results confirm that $21^{\circ} \mathrm{C}$ can be considered as an upper limit temperature for longterm physiological processes in sticklebacks.

\subsubsection{Energy reserves}

As for antioxidant parameters, cadmium at $0.50 \mu \mathrm{g}$. $\mathrm{L}^{-1}$ had no effect on G. aculeatus energy reserves, unlike temperature. In fact, no significant difference in carbohydrate quantities was recorded during the experiment in any treated group as compared to the controls. However, a significant decrease in lipid and protein quantities was observed on day 90 in groups exposed to $21^{\circ} \mathrm{C}$ (exposed to cadmium or not), with a concomitant decrease in stickleback weight and size. Environmental temperature has major effects on fish physiology, and high temperatures accelerate metabolic rates (Wootton, 1984). The peculiarity of ectothermic animals lies in their ability to acclimate to different temperatures in their environment, with maximum and minimum tolerance limits (i.e. their tolerance range). When environmental temperatures are beyond the range of their tolerance limits, they can lead to physiological disturbances and decreased fitness. This situation is called "stress condition". Bioenergetics plays a determining role in living organisms when it comes to stress tolerance (Klepsatel et al., 2016). A balance between energy input and expenditure is required to allow for a sufficient energy supply (aerobic scope of an organism) to cover the cost of basal maintenance as well as other functions (growth, reproduction) (Sokolova, 2013). Under stressful conditions, optimal energy allocation is affected: the aerobic scope is reduced, maintenance and survival take priority while growth and reproduction are stopped, and energy reserves are depleted (Klepsatel et al., 2016). In our study, glycogen levels did not differ between the different groups, while protein and lipid contents decreased after 90 days of exposure. Glycogen stores were not affected by cadmium exposure. This result was not surprising since other parameters (i.e. lipids, proteins) were not affected either, considering the very low cadmium concentration to which sticklebacks were exposed. However, in the "temperature" and "cadmium-temperature" groups, sticklebacks may have kept their glycogen stores for a possible episode of hypoxia in the case of a temperature increase. In some circumstances (e.g. warm vegetated or ice- and snow-covered waters), natural habitats of sticklebacks may become deoxygenated (Wootton, 1994). In addition, it is well known that oxygen consumption in sticklebacks, as in other fish species, rises along with 
temperature (Wootton, 1994; Sollid and Nilsson, 2006). We hypothesised that sticklebacks were genetically programmed to survive in poorly oxygenated habitats. Hence, a rising temperature $\left(21^{\circ} \mathrm{C}\right)$ led to the onset of acclimation mechanisms to prepare sticklebacks to cope with a possible hypoxic period that did not happen because all experimental tanks were continuously oxygenated. As a consequence, glycogen stores were not affected, but protein and lipid reserves were used for gluconeogenesis. Hypoxic periods are characterized by the onset of adaptation mechanisms that allow certain fish species such as carp to be more hypoxia- or anoxia-tolerant (Sollid and Nilsson, 2006). In such conditions, besides gill remodelling to improve oxygen uptake from water, fish increase their liver glycogen reserves (Sollid and Nilsson, 2006; Ballantyne, 2013), and the activity of several gluconeogenic enzymes (De Boeck et al., 1997). Hence, in case of a long hypoxic or anoxic period, the longer the utilization of the glycogen store can be delayed, the greater the chances for fish to survive (Sollid and Nilsson, 2006).

\subsection{Effect on digestive enzymes}

Unlike antioxidant and energy reserve parameters, digestive enzymes showed an interesting sensitivity to cadmium and its combination with temperature. Cadmium alone progressively decreased the activity of the three digestive enzymes significantly only on day 90. The digestive tract is one of the main routes of pollutant absorption, and several authors have reported inhibiting or stimulating effects of organic and inorganic contaminants on the digestive enzymes of aquatic organisms depending on the exposure route and/or the dose (Dedourge-Geffard et al., 2012; Filippov et al., 2013). In our study, the impact on digestive enzymes indicates that cadmium at $0.50 \mu \mathrm{g} . \mathrm{L}^{-1}$ passed through or accumulated in the digestive tract. Zebrafish exposed to cadmium $5 \mu \mathrm{M}$ in water accumulated more cadmium in the gut than in the liver (Vergauwen et al., 2013). Unfortunately, we have no data on cadmium bioaccumulation in the digestive tract to support this information.

Contrary to what most studies on digestive enzyme activities report in the literature, sticklebacks were chronically exposed to a very low cadmium concentration representative of the concentrations found in aquatic environments (IRSN, 2004). In vivo exposure of rosy barb fish (Puntius conchonius) to 48 to $181 \mu \mathrm{g}$. $\mathrm{L}^{-1}$ of mercuric chloride decreased intestinal acid phosphatase (ACP) activity, but increased IAP and lactate dehydrogenase activities (Gill et al., 1990). When exposed to a sublethal concentration of cadmium $\left(6.8 \mathrm{mg}\right.$. $\left.\mathrm{L}^{-1}\right)$ for 30 days, fossil cat (Heteropneustes fossilis) showed inhibition of three intestinal enzymes, i.e. trypsin, IAP, and ACP, while pepsin activity increased in stomach (Sastry and Gupta, 1979). Moreover, amylase and protease activities were inhibited after $60 \mathrm{~min}$ of in vitro exposure to 5, 25, and 
$50 \mathrm{mg} . \mathrm{L}^{-1}$ of cadmium, and carbohydrase activity was reduced after 60 days of in vivo exposure to $5 \mathrm{mg} . \mathrm{L}^{-1}$ of cadmium in Mozambique tilapias (Oreochromis mossambicus) (Golovanova et al., 1994). The inhibitory effect of heavy metals on enzymes can operate through two mechanisms: (i) a direct effect, i.e. a modification of the spatial conformation of the enzyme through the interaction of the pollutant with essential elements such as $\mathrm{Ca}^{2+}$ ions (in the case of cadmium for example), resulting in altered enzyme activity, and (ii) an indirect effect via interactions with synthesis and/or secretion mechanisms (Dedourge-Geffard et al., 2012).

Temperature also impacted G. aculeatus digestive enzymes (trypsin, IAP, amylase), and aggravated the effect of cadmium when the two stresses were combined. The effect of temperature (with or without cadmium exposure) was recorded earlier, i.e. from day 15, as compared to the single effect of cadmium. Fish are heavily influenced by environmental temperature because they are heterothermic animals. When we exposed sticklebacks to an elevated temperature $\left(21^{\circ} \mathrm{C}\right)$ in the absence of cadmium, trypsin and IAP activities decreased from day 15, while amylase activity remained stable throughout the experiment. These results are not consistent with the literature. Numerous authors have indeed investigated the effect of temperature on fish digestive enzymes. In general, when temperature increases, digestive enzyme activities increase too (Hofer, 1979a, 1979b; Kuz'mina et al., 2002; Bowyer et al., 2014).

Two hypotheses can be proposed to explain why the inhibiting effect of cadmium on digestive enzymes was promoted at $21^{\circ} \mathrm{C}$. The first hypothesis is that the elevated temperature was responsible for a high rate of cadmium accumulation in the gut (contrary to what was observed in liver), leading to enhanced effects of cadmium on digestive enzymes. The second hypothesis is that sticklebacks raised at $21^{\circ} \mathrm{C}$ produced new IAP, trypsin and amylase isoenzymes, as reported in other fish species as an acclimation mechanism to temperature (Gelman et al., 2008). These-new isoenzymes were more vulnerable to the effects of cadmium than those produced at $16^{\circ} \mathrm{C}$.

Despite the very low cadmium concentration to which sticklebacks were exposed, and compared to classical biomarkers of metal exposure, G. aculeatus digestive enzymes responded rapidly. Therefore, they appear as an interesting early warning tool to reflect the health status of organisms and the chemical quality of the surrounding water. The highest sensitivity of digestive enzyme responses suggests that these measurements are relevant for assessing the effects of water quality on the digestive capacity of G. aculeatus. The interest of these enzymes as potential biomarkers of the health status of organisms and of the chemical quality of aquatic 
ecosystems is particularly true for trypsin and IAP activities. These results are in agreement with Lemieux et al. (1999) and Rungruangsak-Torrissen et al. (2006), who reported that these enzymes are limiting factors for fish growth. However, further studies are necessary to more precisely characterise the potential effects of other contaminant families (e.g. PAHs, pesticides) and abiotic and biotic parameters on the above-described enzyme activities before using them as biomarkers because they can be confounding factors.

\section{Conclusions}

This study aimed to (i) evaluate the combined effects of 90 days of exposure to an environmental dose of cadmium and an elevated water temperature $\left(21{ }^{\circ} \mathrm{C}\right)$ on threespine stickleback physiology, and (ii) to evaluate the interest of stickleback digestive enzymes (IAP, trypsin, amylase) as new biomarkers. Sticklebacks were exposed to a very low cadmium concentration $\left(0.50 \mu \mathrm{g} . \mathrm{L}^{-1}\right)$, which is below the freshwater chronic criterion (CCC) of $0.72 \mu \mathrm{g}$. $\mathrm{L}^{-1}$ recommended by the US Environmental Protection Agency (EPA).

On the one hand, a temperature of $21{ }^{\circ} \mathrm{C}$, which is cogent with a predicted global temperature rise of $1.8-4.0^{\circ} \mathrm{C}$, impacted G. aculeatus antioxidant parameters and protein and lipid energy reserves after a long exposure period. In the same way, stickleback growth was limited under the effect of the high temperature, and reproduction also seemed to be impacted. This could have rather serious consequences on stickleback populations in a long-term climate warming context. These results suggest that $21{ }^{\circ} \mathrm{C}$ is an upper tolerance limit for long-term physiology processes in threespine sticklebacks.

On the other hand, cadmium alone at the very low concentration of $0.50 \mu \mathrm{g}$. $\mathrm{L}^{-1}$ had no effect on any parameters, except digestive enzymes that were negatively impacted after 90 days of exposure. This effect on digestive enzymes was aggravated and recorded earlier under the higher temperature. Compared to conventional measurements of the effects of metals on fish physiology (i.e. biometric parameters, antioxidant biomarkers, energy reserves), G. aculeatus digestive enzymes responded rapidly and therefore appear as interesting early warning tools for reflecting the health status of organisms.

Reproduction is one of the most important biological functions in living organisms. It would therefore be of great interest to investigate the relationship between early impairment of G. aculeatus digestive enzyme activities and the reproductive function. 
Acknowledgments. This work was partly supported by the University of Reims

Champagne-Ardenne and the financial support of the P190 Ecotoxicology Program of the

French Ministry for Ecology and Sustainable Development. The authors are grateful to

Mrs. Annie BUCHWALTER for editing English language.

\section{References}

Acharia, K., Lal, B., Singh, T.P., 2014. Season-dependent effect of thermopulse on gonadal recrudescence in the female catfish, Clarias batrachus.

Almeida, J.A., Novelli, E.L.B., Dal Pai Silva, M., Alves Júnior, R., 2001. Environmental cadmium exposure and metabolic responses of the Nile tilapia, Oreochromis niloticus. Environ. Pollut. 114, 169-175. https://doi.org/10.1016/S0269-7491(00)00221-9

Andersen, Ø., Frantzen, M., Rosland, M., Timmerhaus, G., Skugor, A., Krasnov, A., 2015. Effects of crude oil exposure and elevated temperature on the liver transcriptome of polar cod (Boreogadus saida). Aquat. Toxicol. 165, 9-18. https://doi.org/10.1016/j.aquatox.2015.04.023

Andres, S., Ribeyre, F., Tourencq, J.N., Boudou, A., 2000. Interspecific comparison of cadmium and zinc contamination in the organs of four fish species along a polymetallic pollution gradient (Lot River, France). Sci. Total Environ. 248, 11-25. https://doi.org/10.1016/S0048-9697(99)00477-5

Authman, M.M., Zaki, M.S., Khallaf, E.A., Abbas, H.H., 2015. Use of fish as bio-indicator of the effects of heavy metals pollution. J. Aquac. Res. Dev. 6, 1.

Ballantyne, J.S., 2013. Membranes and Metabolism, in: Evans, D.H., Claiborne, J.B., Currie, S. (Eds.), The Physiology of Fishes, Fourth Edition. Taylor \& Francis.

Bowyer, J.N., Booth, M.A., Qin, J.G., D’Antignana, T., Thomson, M.J.S., Stone, D.A.J., 2014. Temperature and dissolved oxygen influence growth and digestive enzyme activities of yellowtail kingfish Seriola lalandi (Valenciennes, 1833). Aquac. Res. 45, 2010-2020. https://doi.org/10.1111/are.12146

Bradford, M.M., 1976. A rapid and sensitive method for the quantitation of microgram quantities of protein utilizing the principle of protein-dye binding. Anal. Biochem. 72, 248-254. https://doi.org/10.1016/0003-2697(76)90527-3

Caruso, G., De Pasquale, F., Mita, D.G., Micale, V., 2016. Digestive enzymatic patterns as possible biomarkers of endocrine disruption in the red mullet (Mullus barbatus): A preliminary investigation. Mar. Pollut. Bull. 105, 37-42.

https://doi.org/10.1016/j.marpolbul.2016.03.005

CAS, 2017. Homepage of the Chemical Abstracts Service [WWW Document]. URL (accessed 8.8.17).

Cazenave, J., Bistoni, M. de los A., Pesce, S.F., Wunderlin, D.A., 2006. Differential detoxification and antioxidant response in diverse organs of Corydoras paleatus experimentally exposed to microcystin-RR. Aquat. Toxicol. 76, 1-12. https://doi.org/10.1016/j.aquatox.2005.08.011

Charron, L., Geffard, O., Chaumot, A., Coulaud, R., Queau, H., Geffard, A., DedourgeGeffard, O., 2013. Effect of water quality and confounding factors on digestive enzyme activities in Gammarus fossarum. Environ. Sci. Pollut. Res. 20, 9044-9056. https://doi.org/10.1007/s11356-013-1921-5 
Cheng, Y.-S., Zheng, Y., VanderGheynst, J.S., 2011. Rapid Quantitative Analysis of Lipids Using a Colorimetric Method in a Microplate Format. Lipids 46, 95-103. https://doi.org/10.1007/s11745-010-3494-0

Cossu, C., Doyotte, A., Babut, M., Exinger, A., Vasseur, P., 2000. Antioxidant Biomarkers in Freshwater Bivalves, Unio tumidus, in Response to Different Contamination Profiles of Aquatic Sediments. Ecotoxicol. Environ. Saf. 45, 106-121. https://doi.org/10.1006/eesa.1999.1842

De Boeck, G., Vlaeminck, A., Blust, R., 1997. Effects of sublethal copper exposure on copper accumulation, food consumption, growth, energy stores, and nucleic acid content in common carp. Arch. Environ. Contam. Toxicol. 33, 415-422.

De Coen, W., Janssen, C., 1997. The use of biomarkers in Daphnia magna toxicity testing II. Digestive enzyme activity in Daphnia magna exposed to sublethal concentrations of cadmium, chromium and mercury. Chemosphere 35, 1053-1067. https://doi.org/10.1016/S0045-6535(97)00172-0

De Coen, W.M., Janssen, C.R., 2003. A multivariate biomarker-based model predicting population-level responses of Daphnia magna. Environ. Toxicol. Chem. 22, 21952201. https://doi.org/10.1897/02-223

Dedourge-Geffard, O., Palais, F., Biagianti-Risbourg, S., Geffard, O., Geffard, A., 2009. Effects of metals on feeding rate and digestive enzymes in Gammarus fossarum: An in situ experiment. Chemosphere 77, 1569-1576. https://doi.org/10.1016/j.chemosphere.2009.09.042

Dedourge-Geffard, O., Palais, F., Geffard, A., Amiard-Triquet, C., 2012. Origin of energy metabolism impairments. Ecol. Biomarkers-indicators Ecotoxicological Eff. CRC Press Taylor Francis Group Boca Raton 279-306.

Dickson, G.W., Giesy, J.P., Briese, L.A., 1982. The effect of chronic cadmium exposure on phosphoadenylate concentrations and adenylate energy charge of gills and dorsal muscle tissue of crayfish. Environ. Toxicol. Chem. 1, 147-156. https://doi.org/10.1002/etc.5620010206

Farombi, E., Adelowo, O., Ajimoko, Y., 2007. Biomarkers of oxidative stress and heavy metal levels as indicators of environmental pollution in African cat fish (Clarias gariepinus) from Nigeria Ogun River. Int. J. Environ. Res. Public. Health 4, 158-165.

Ferrari, L., Eissa, B.L., Salibián, A., 2011. Energy balance of juvenile Cyprinus carpio after a short-term exposure to sublethal water-borne cadmium. Fish Physiol. Biochem. 37, 853-862. https://doi.org/10.1007/s10695-011-9483-2

Figueiredo-Fernandes, A., Fontaínhas-Fernandes, A., Peixoto, F., Rocha, E., Reis-Henriques, M., 2006. Effects of gender and temperature on oxidative stress enzymes in Nile tilapia Oreochromis niloticus exposed to paraquat. Pestic. Biochem. Physiol. 85, $97-$ 103.

Filippov, A., Golovanova, I., Aminov, A., 2013. Effects of organic pollutants on fish digestive enzymes: A review. Inland Water Biol. 6, 155-160.

Foran, C.M., Peterson, B.N., Benson, W.H., 2002. Influence of parental and developmental cadmium exposure on endocrine and reproductive function in Japanese medaka (Oryzias latipes). Comp. Biochem. Physiol. Part C Toxicol. Pharmacol. 133, 345-354. https://doi.org/10.1016/S1532-0456(02)00128-X

Forbes, V.E., Palmqvist, A., Bach, L., 2006. The use and misuse of biomarkers in ecotoxicology. Environ. Toxicol. Chem. 25, 272-280. https://doi.org/10.1897/05257R. 1

Garcia-Carreño, F.L., Haard, N.F., 1993. Characterization of proteinase classes in langostilla (Pleuroncodes planipes) and crayfish (Pacifastacus astacus) extracts. J. Food Biochem. 17, 97-113. https://doi.org/10.1111/j.1745-4514.1993.tb00864.x 
Geffard, A., Sartelet, H., Garric, J., Biagianti-Risbourg, S., Delahaut, L., Geffard, O., 2010. Subcellular compartmentalization of cadmium, nickel, and lead in Gammarus fossarum: Comparison of methods. Chemosphere 78, 822-829. https://doi.org/10.1016/j.chemosphere.2009.11.051

Gelman, A., Kuz'mina, V., Drabkin, V., Glatman, L., 2008. Temperature Adaptation of Digestive Enzymes in Fish, in: Feeding and Digestive Functions in Fishes. Taylor \& Francis Group, LLC, pp. 155-225. https://doi.org/10.1201/b10749-6

Gill, T.S., Tewari, H., Pande, J., 1990. Use of the fish enzyme system in monitoring water quality: effects of mercury on tissue enzymes. Comp. Biochem. Physiol. Part C Comp. Pharmacol. 97, 287-292.

Golovanova, I.L., Chuiko, G.M., Pavlov, D.F., 1994. Effects of cadmium, naphthalene, and DDVP on gut carbohydrases activity in bream (Abramis brama L.) and Mozambique tilapia (Oreochromis mossambicus Peters). Bull. Environ. Contam. Toxicol. 52, 338345. https://doi.org/10.1007/bf00197818

Guardiola, F.A., Dioguardi, M., Parisi, M.G., Trapani, M.R., Meseguer, J., Cuesta, A., Cammarata, M., Esteban, M.A., 2015. Evaluation of waterborne exposure to heavy metals in innate immune defences present on skin mucus of gilthead seabream (Sparus aurata). Fish Shellfish Immunol. 45, 112-123. https://doi.org/10.1016/j.fsi.2015.02.010

Guderley, H.E., 1994. Physioclogical ecology and evolution of the threespine stickleback, in: Bell, M.A., Foster, S.A. (Eds.), The Evolutionary Biology of the Threespine Stickleback. Oxford University Press, New York, pp. 85-113.

Hallare, A.V., Schirling, M., Luckenbach, T., Köhler, H.R., Triebskorn, R., 2005. Combined effects of temperature and cadmium on developmental parameters and biomarker responses in zebrafish (Danio rerio) embryos. J. Therm. Biol. 30, 7-17. https://doi.org/10.1016/j.jtherbio.2004.06.002

Hamilton, P.B., Cowx, I.G., Oleksiak, M.F., Griffiths, A.M., Grahn, M., Stevens, J.R., Carvalho, G.R., Nicol, E., Tyler, C.R., 2015. Population-level consequences for wild fish exposed to sublethal concentrations of chemicals-a critical review. Fish Fish.

Heugens, E.H.W., Hendriks, A.J., Dekker, T., Straalen, N.M. van, Admiraal, W., 2001. A Review of the Effects of Multiple Stressors on Aquatic Organisms and Analysis of Uncertainty Factors for Use in Risk Assessment. Crit. Rev. Toxicol. 31, 247-284. https://doi.org/10.1080/20014091111695

Hofer, R., 1979a. The adaptation of digestive enzymes to temperature, season and diet in roach, Rutilus rutilus L. and rudd Scardinius erythrophthalmus L. 1. Amylase. J. Fish Biol. 14, 565-572. https://doi.org/10.1111/j.1095-8649.1979.tb03556.x

Hofer, R., 1979b. The adaptation of digestive enzymes to temperature, season and diet in roach, Rutilus rutilus and rudd Scardinius erythrophthalmus; Proteases. J. Fish Biol. 15, 373-379. https://doi.org/10.1111/j.1095-8649.1979.tb03619.x

Hooper, M.J., Ankley, G.T., Cristol, D.A., Maryoung, L.A., Noyes, P.D., Pinkerton, K.E., 2013. Interactions between chemical and climate stressors: A role for mechanistic toxicology in assessing climate change risks. Environ. Toxicol. Chem. 32, 32-48. https://doi.org/10.1002/etc.2043

Hovel, R.A., Beauchamp, D.A., Hansen, A.G., Sorel, M.H., 2015. Development of a Bioenergetics Model for the Threespine Stickleback. Trans. Am. Fish. Soc. 144, 1311-1321. https://doi.org/10.1080/00028487.2015.1079554

Huntingford, F.A., Chellappa, S., Taylor, A.C., Strang, R.H.C., 2001. Energy reserves and reproductive investment in male three-spined sticklebacks, Gasterosteus aculeatus. Ecol. Freshw. Fish 10, 111-117. https://doi.org/10.1034/j.1600-0633.2001.100206.x 
IPCC, 2013. Climate change_The Physical Science Basis: the fifth assessment report of the intergovernmental panel on climate change (IPCC).

IRSN, 2004. FICHE RADIONUCLÉIDE cadmium 109 et environnement. France.

Jordan, C.M., Garside, E., 1972. Upper lethal temperatures of threespine stickleback, Gasterosteus aculeatus (L.), in relation to thermal and osmotic acclimation, ambient salinity, and size. Can. J. Zool. 50, 1405-1411.

Junge, W., Wortmann, W., Wilke, B., Waldenström, J., Kurrle-Weittenhiller, A., Finke, J., Klein, G., 2001. Development and evaluation of assays for the determination of total and pancreatic amylase at $37^{\circ} \mathrm{C}$ according to the principle recommended by the IFCC. Clin. Biochem. 34, 607-615. https://doi.org/10.1016/S0009-9120(01)00278-8

Klepsatel, P., Gáliková, M., Xu, Y., Kühnlein, R.P., 2016. Thermal stress depletes energy reserves in Drosophila. Sci. Rep. 6.

Kuz'mina, V., Golovanova, I., Kovalenko, E., 2002. Separate and combined effects of cadmium, temperature, and $\mathrm{pH}$ on digestive enzymes in three freshwater teleosts. Bull. Environ. Contam. Toxicol. 69, 302-308.

Leloutre, C., Péry, A.R.R., Porcher, J.-M., Beaudouin, R., 2016. A bioenergetics model of the entire life cycle of the three-spined stickleback, gasterosteus aculeatus. Ecol. Freshw. Fish n/a-n/a. https://doi.org/10.1111/eff.12329

Lemieux, H., Blier, P., Dutil, J.-D., 1999. Do digestive enzymes set a physiological limit on growth rate and food conversion efficiency in the Atlantic cod (Gadus morhua)? Fish Physiol. Biochem. 20, 293-303. https://doi.org/10.1023/a:1007791019523

Levesque, H.M., Moon, T.W., Campbell, P.G.C., Hontela, A., 2002. Seasonal variation in carbohydrate and lipid metabolism of yellow perch (Perca flavescens) chronically exposed to metals in the field. Aquat. Toxicol. 60, 257-267. https://doi.org/10.1016/S0166-445X(02)00012-7

Li, A.J., Leung, P.T.Y., Bao, V.W.W., Yi, A.X.L., Leung, K.M.Y., 2014. Temperaturedependent toxicities of four common chemical pollutants to the marine medaka fish, copepod and rotifer. Ecotoxicology 23, 1564-1573. https://doi.org/10.1007/s10646014-1297-4

Madeira, D., Narciso, L., Cabral, H., Vinagre, C., Diniz, M., 2013. Influence of temperature in thermal and oxidative stress responses in estuarine fish. Comp. Biochem. Physiol.

A. Mol. Integr. Physiol. 166, 237-243.

Munday, P.L., Kingsford, M.J., O’Callaghan, M., Donelson, J.M., 2008. Elevated temperature restricts growth potential of the coral reef fish Acanthochromis polyacanthus. Coral Reefs 27, 927-931. https://doi.org/10.1007/s00338-008-0393-4

OECD, 2012. Test No. 211: Daphnia magna Reproduction Test. OECD Publishing.

Olsvik, P.A., Søfteland, L., Hevrøy, E.M., Rasinger, J.D., Waagbø, R., 2016. Fish preacclimation temperature only modestly affects cadmium toxicity in Atlantic salmon hepatocytes. J. Therm. Biol. 57, 21-34. https://doi.org/10.1016/j.jtherbio.2016.02.003

Panteghini, M.M.D., Bais, R., 2008. Ezymes, in: Burtis, C.A.., Ashwood, E.R., D, B.D.E.M. (Eds.), Tietz Fundamentals of Clinical Chemistry. Saunders ELSEVIER, pp. 317-336.

Paoletti, F., Aldinucci, D., Mocali, A., Caparrini, A., 1986. A sensitive spectrophotometric method for the determination of superoxide dismutase activity in tissue extracts. Anal. Biochem. 154, 536-541. https://doi.org/10.1016/0003-2697(86)90026-6

Perera, P., Kodithuwakku, S., Sundarabarathy, T., Edirisinghe, U., 2015. Bioaccumulation of cadmium in freshwater fish: an environmental perspective. Insight Ecol 4, 1-12.

Pi, J., Li, X., Zhang, T., Li, D., 2016. Effects of Acute Exposure to Sublethal Waterborne Cadmium on Energy Homeostasis in Silver Carp (Hypophthalmichthys molitrix). Bull. Environ. Contam. Toxicol. 97, 497-503. https://doi.org/10.1007/s00128-0161896-2 
Pretto, A., Loro, V.L., Morsch, V.M., Moraes, B.S., Menezes, C., Santi, A., Toni, C., 2014. Alterations in carbohydrate and protein metabolism in silver catfish (Rhamdia quelen) exposed to cadmium. Ecotoxicol. Environ. Saf. 100, 188-192. https://doi.org/10.1016/j.ecoenv.2013.11.004

Reynaud, S., Deschaux, P., 2006. The effects of polycyclic aromatic hydrocarbons on the immune system of fish: A review. Aquat. Toxicol. 77, 229-238. https://doi.org/10.1016/j.aquatox.2005.10.018

Rungruangsak-Torrissen, K., Moss, R., Andresen, L.H., Berg, A., Waagbø, R., 2006. Different expressions of trypsin and chymotrypsin in relation to growth in Atlantic salmon (Salmo salar L.). Fish Physiol. Biochem. 32, 7-23. https://doi.org/10.1007/s10695-005-0630-5

Sanchez, W., Aït-Aïssa, S., Palluel, O., Ditche, J.-M., Porcher, J.-M., 2007. Preliminary investigation of multi-biomarker responses in three-spined stickleback (Gasterosteus aculeatus L.) sampled in contaminated streams. Ecotoxicology 16, 279-287. https://doi.org/10.1007/s10646-006-0131-z

Sanchez, W., Palluel, O., Meunier, L., Coquery, M., Porcher, J.-M., Aït-Aïssa, S., 2005. Copper-induced oxidative stress in three-spined stickleback: relationship with hepatic metal levels. Environ. Toxicol. Pharmacol. 19, 177-183. https://doi.org/10.1016/j.etap.2004.07.003

Sancho, E., Ferrando, M.D., Fernández, C., Andreu, E., 1998. Liver Energy Metabolism ofAnguilla anguillaafter Exposure to Fenitrothion. Ecotoxicol. Environ. Saf. 41, 168 175. https://doi.org/10.1006/eesa.1998.1689

Sastry, K.V., Gupta, P.K., 1979. The effect of cadmium on the digestive system of the teleost fish, Heteropneustes fossilis. Environ. Res. 19, 221-230. https://doi.org/10.1016/00139351(79)90050-1

Sfakianakis, D.G., Renieri, E., Kentouri, M., Tsatsakis, A.M., 2015. Effect of heavy metals on fish larvae deformities: A review. Environ. Res. 137, 246-255. https://doi.org/10.1016/j.envres.2014.12.014

Soengas, J., Agra-Lago, M., Carballo, B., Andres, M., Veira, J., 1996. Effect of an acute exposure to sublethal concentrations of cadmium on liver carbohydrate metabolism of Atlantic salmon (Salmo salar). Bull. Environ. Contam. Toxicol. 57, 625-631.

Sokolova, I.M., 2013. Energy-Limited Tolerance to Stress as a Conceptual Framework to Integrate the Effects of Multiple Stressors. Integr. Comp. Biol. 53, 597-608. https://doi.org/10.1093/icb/ict028

Sollid, J., Nilsson, G.E., 2006. Plasticity of respiratory structures - Adaptive remodeling of fish gills induced by ambient oxygen and temperature. Respir. Physiol. Neurobiol. 154, 241-251. https://doi.org/10.1016/j.resp.2006.02.006

US EPA, U.E.P.A., 2016. Recommended aquatic life ambient water quality criteria for cadmium.EPA HQ/OW/2015/0753. FRL 9944-46-OW. Document no. 2016-07647. Washington, DC.

Van Handel, E., 1965. Microseparation of glycogen, sugars, and lipids. Anal. Biochem. 11, 266-271. https://doi.org/10.1016/0003-2697(65)90014-X

Vandeputte, C., Guizon, I., Genestie-Denis, I., Vannier, B., Lorenzon, G., 1994. A microtiter plate assay for total glutathione and glutathione disulfide contents in cultured/isolated cells: performance study of a new miniaturized protocol. Cell Biol. Toxicol. 10, 415421. https://doi.org/10.1007/bf00755791

Vergauwen, L., Hagenaars, A., Blust, R., Knapen, D., 2013. Temperature dependence of longterm cadmium toxicity in the zebrafish is not explained by liver oxidative stress: Evidence from transcript expression to physiology. Aquat. Toxicol. 126, 52-62. https://doi.org/10.1016/j.aquatox.2012.10.004 
Vinagre, C., Madeira, D., Narciso, L., Cabral, H.N., Diniz, M., 2012. Effect of temperature on oxidative stress in fish: Lipid peroxidation and catalase activity in the muscle of juvenile seabass, Dicentrarchus labrax. Ecol. Indic. 23, 274-279. https://doi.org/10.1016/j.ecolind.2012.04.009

Wang, T., Long, X., Cheng, Y., Liu, Z., Yan, S., 2015. A Comparison Effect of Copper Nanoparticles versus Copper Sulphate on Juvenile Epinephelus coioides: Growth Parameters, Digestive Enzymes, Body Composition, and Histology as Biomarkers. Int. J. Genomics 2015, 783021. https://doi.org/10.1155/2015/783021

Watanabe, N., Kamei, S., Ohkubo, A., Yamanaka, M., Ohsawa, S., Makino, K., Tokuda, K., 1986. Urinary protein as measured with a pyrogallol red-molybdate complex, manually and in a Hitachi 726 automated analyzer. Clin. Chem. 32, 1551-1554.

Weston, D.P., You, J., Harwood, A.D., Lydy, M.J., 2009. Whole sediment toxicity identification evaluation tools for pyrethroid insecticides: III. Temperature manipulation. Environ. Toxicol. Chem. 28, 173-180. https://doi.org/10.1897/08-143.1

Wootton, R., 1994. Energy allocation in the threespine stickleback, in: Bell, M.A., Foster, S.A. (Eds.), The Evolutionary Biology of the Threespine Stickleback. Oxford University Press, New York, pp. 114-143.

Wootton, R., 1984. A functional biology of sticklebacks. Croom Helm.

Zheng, J.-L., Yuan, S.-S., Wu, C.-W., Li, W.-Y., 2016. Chronic waterborne zinc and cadmium exposures induced different responses towards oxidative stress in the liver of zebrafish. Aquat. Toxicol. 177, 261-268.

https://doi.org/10.1016/j.aquatox.2016.06.001 